\title{
Star formation efficiency in galaxy interactions and mergers: a statistical study
}

\author{
P. Di Matteo, F. Combes, A.-L.Melchior, and B. Semelin
}

\begin{abstract}
Observatoire de Paris, LERMA, 61 Avenue de L'Observatoire, 75014 Paris, France
e-mail: [paola.dimatteo; francoise.combes; anne-laure.melchior; benoit.semelin]@obspm.fr
\end{abstract}

Received 18 December 2006 / Accepted 5 Mars 2007

\begin{abstract}
We investigate the enhancement of star formation efficiency in galaxy interactions and mergers by numerical simulations of several hundred galaxy collisions. All morphological types along the Hubble sequence are considered in the initial conditions of the two colliding galaxies, with varying bulge-to-disk ratios and gas mass fractions. Different types of orbits are simulated, direct and retrograde, according to the initial relative energy and impact parameter, and the resulting star formation history is compared to that occuring in the two galaxies when they are isolated. Our principal results are (1) retrograde encounters have greater star formation efficiency (SFE) than direct encounters, (2) the amount of gas available in the galaxy is not the main parameter governing the SFE in the burst phase, (3) there is a negative correlation between the amplitude of the star forming burst and the tidal forces exerted per unit of time, which is due to the large amount of gas dragged outside the galaxy by tidal tails in strong interactions, (4) globally, the Kennicutt-Schmidt law is seen to apply statistically for isolated galaxies, interacting pairs and mergers, (5) enhanced star formation occurs essentially in nuclear starbursts, triggered by inward gas flows driven by non-axisymmetries in the galaxy disks. Direct encounters develop more pronounced asymmetries than retrograde ones. Based on these statistical results we derive general laws for the enhancement of star formation in galaxy interactions and mergers, as a function of the main parameters of the encounter.
\end{abstract}

Key words. galaxies: evolution - galaxies: interactions - galaxies: starburst - methods: numerical

\section{Introduction}

Theories about the formation and evolution of galaxies have undergone drastic changes in the last decade. Impressive progress in the observations of galaxies at high and intermediate redshift now put constraints directly on galaxy evolution (Steidel et al. 1999; Le Fèvre et al. 2000; Cimatti et al. 2002; Flores et al. 2004). The star formation history can be estimated (Madau et al. 1996; Lilly et al. 1996), and the confrontation with numerical simulations helps us to discover the actual mechanisms of mass assembly and transformation of gas into stars (Kauffmann \& Charlot 1998; Somerville et al. 2001). Models are built, on the one hand, through cosmological $N$-body simulations (e.g. Springel \& Hernquist 2003; Sommer-Larsen et al. 2003; Governato et al. 2004), although they generally lack the spatial resolution at galaxy scales that would enable them to resolve star-formation related processes in a realistic way; on the other hand, simulations are then complemented by semi-analytic computations, where galaxy evolution is treated with recipes taking into account all important processes, including hierarchical merging and star formation (Devriendt \& Guiderdoni 2000; Kauffmann \& Haehnelt 2000).

In both cases, it is necessary to study in more detail the star formation efficiency at the galaxy scale, to calibrate the physical recipes: star formation rate and feedback processes have been computed on isolated and merging galaxies, and a large variety of results have been obtained (Mihos \& Hernquist 1994a,b, 1996; Springel 2000; Tissera et al. 2002; Meza et al. 2003; Kapferer et al. 2005; Cox et al. 2006). All studies demonstrated that galaxy collisions trigger star formation, and that the initial disk stability is the main parameter affecting the star formation sequence: late-type galaxies without bulge are more prone to violent bar instability during an encounter, which drives the internal gas towards the galaxy center to trigger a nuclear starburst (Mihos \& Hernquist 1996). However, the availability of gas in the interacting galaxies is also one of the more constraining parameters, as well as the gas physics adopted (Cox et al. 2004).

The amount of star formation triggered due to the interaction is a fundamental parameter required by semi-analytical simulations and one that depends very much on models adopted for star formation rate and feedback. We propose here to investigate this problem statistically, simulating all galaxy types along the Hubble sequence, and exploring all physical parameters for the interactions, mass ratios, geometrical orbital parameters, etc.. in order to get an insight into the global phenomena and their range of variations. In this first paper the numerical method and galaxy models are presented and star formation evolution involving giant-like galaxies are presented and discussed. A wider range of interactions and mergers involving the whole mass spectrum of galaxies will be considered in a companion paper. Galaxies are initiated at $z=0$, while simulations involving galaxies at any redshift will be studied in a later research.

The layout of the paper is as follows. In Sect. 2, the galaxy models adopted (Sect. 2.1) and the initial orbital parameters (Sect. 2.2) are described; the numerical code is described in Sect. 3; in Sect. 4 the main results are presented, in particular those related to the evolution of the star formation rate during the different phases of interaction, its dependency on the total gas amount in the galaxies and on the main orbital parameters of the encounter. Finally, in Sect. 5 we analyze and discuss the interaction-starburst connection and in Sect. 6 the main conclusions of this research are presented. 


\section{Initial conditions}

\subsection{Galaxy models: moving along the Hubble sequence}

Aiming at exploiting a large set of interactions, involving galaxies of all morphologies from ellipticals to late-type spirals, the galaxy models adopted consist of a spherical dark-matter halo, which may or may not contain a stellar and a gaseous disk and, optionally, a central bulge.

For each galaxy type ${ }^{1}$, the halo and the optional bulge are modeled as a Plummer sphere (Binney \& Tremaine 1987, p. 42), with characteristic masses $M_{\mathrm{B}}$ and $M_{\mathrm{H}}$ and characteristic radii $r_{\mathrm{B}}$ and $r_{\mathrm{H}}$. Their densities are given, respectively, by:

$\rho_{\mathrm{H}}(r)=\left(\frac{3 M_{\mathrm{H}}}{4 \pi r_{\mathrm{H}}^{3}}\right)\left(1+\frac{r^{2}}{r_{\mathrm{H}}^{2}}\right)^{-5 / 2}$

and

$\rho_{\mathrm{B}}(r)=\left(\frac{3 M_{\mathrm{B}}}{4 \pi r_{\mathrm{B}}{ }^{3}}\right)\left(1+\frac{r^{2}}{r_{\mathrm{B}}^{2}}\right)^{-5 / 2}$.

The stellar and gaseous disks follow a Miyamoto-Nagai density profile (Binney \& Tremaine 1987, p. 44):

$$
\begin{aligned}
& \rho_{*}(R, z)=\left(\frac{h_{*}^{2} M_{*}}{4 \pi}\right) \\
& \times \frac{a_{*} R^{2}+\left(a_{*}+3 \sqrt{z^{2}+h_{*}^{2}}\right)\left(a_{*}+\sqrt{z^{2}+h_{*}^{2}}\right)^{2}}{\left[a_{*}^{2}+\left(a_{*}+\sqrt{z^{2}+h_{*}^{2}}\right)^{2}\right]^{5 / 2}\left(z^{2}+h_{*}^{2}\right)^{3 / 2}} \\
& \rho_{\mathrm{g}}(R, z)=\left(\frac{h_{\mathrm{g}}{ }^{2} M_{\mathrm{g}}}{4 \pi}\right) \\
& \times \frac{a_{\mathrm{g}} R^{2}+\left(a_{\mathrm{g}}+3 \sqrt{z^{2}+{h_{\mathrm{g}}}^{2}}\right)\left(a_{\mathrm{g}}+\sqrt{z^{2}+h_{\mathrm{g}}{ }^{2}}\right)^{2}}{\left[a_{\mathrm{g}}^{2}+\left(a_{\mathrm{g}}+\sqrt{z^{2}+h_{\mathrm{g}}^{2}}\right)^{2}\right]^{5 / 2}\left(z^{2}+h_{\mathrm{g}}{ }^{2}\right)^{3 / 2}},
\end{aligned}
$$

with masses $M_{*}$ and $M_{\mathrm{g}}$ and vertical and radial scale lengths given, respectively, by $h_{*}$ and $a_{*}$, and $h_{\mathrm{g}}$ and $a_{\mathrm{g}}$. Moving along the Hubble sequence, from giant-like ellipticals (gE0) to giantlike $\mathrm{Sd}$ spirals $(\mathrm{gSd})$, the mass of the central spheroid varies from $M_{\mathrm{B}}=1.6 \times 10^{11} M_{\odot}$ for a $\mathrm{gE0}$ to $M_{\mathrm{B}}=0$ for a $\mathrm{gSd}$, while the gas mass $M_{\mathrm{g}}$, absent in the case of a $\mathrm{gE} 0$, increases from $9.2 \times$ $10^{9} M_{\odot}$ in a gSa to $1.7 \times 10^{10} M_{\odot}$ for a gSd (see Table 1 for a complete list of all the parameters in Eqs. (1) to (4) and Fig. 1 for a representation of our galaxy sequence).

The initial rotation curves for spiral galaxies are shown in Fig. 2. In accordance with observations (Roberts \& Haynes 1994), for a fixed distance from the galaxy center, the value of $V_{\text {rot }}$ is higher for early type systems than for late type ones, indicating a decrease in the enclosed mass content. Aiming at investigating interactions between giant-like galaxies, the mass ratios of the interacting systems is always of order unity. In the next future, we will exploit collisions involving galaxies across the whole mass spectrum.

\footnotetext{
${ }^{1}$ Hereafter we will adopt the following nomenclature for the different morphological types: gE0 for giant-like ellipticals, gSa for giantlike Sa spirals, gSb for giant-like Sbc spirals and gSd for giant-like Sd spirals.
}

Table 1. Galaxy parameters. The bulge and the halo are modelled as Plummer spheres, with characteristic masses $M_{\mathrm{B}}$ and $M_{\mathrm{H}}$ and charac-

\begin{tabular}{|c|c|c|c|c|}
\hline & gE0 & $\mathrm{gSa}$ & $\mathrm{gSb}$ & $\mathrm{gSd}$ \\
\hline$M_{\mathrm{B}}\left[2.3 \times 10^{9} M_{\odot}\right]$ & 70 & 10 & 5 & 0 \\
\hline$M_{\mathrm{H}}\left[2.3 \times 10^{9} M_{\odot}\right]$ & 30 & 50 & 75 & 75 \\
\hline$M_{*}\left[2.3 \times 10^{9} M_{\odot}\right]$ & 0 & 40 & 20 & 25 \\
\hline$M_{\mathrm{g}} / M_{*}$ & 0 & 0.1 & 0.2 & 0.3 \\
\hline$r_{\mathrm{B}}[\mathrm{kpc}]$ & 4 & 2 & 1 & - \\
\hline$r_{\mathrm{H}}[\mathrm{kpc}]$ & 7 & 10 & 12 & 15 \\
\hline$a_{*}[\mathrm{kpc}]$ & - & 4 & 5 & 6 \\
\hline$h_{*}[\mathrm{kpc}]$ & - & 0.5 & 0.5 & 0.5 \\
\hline$a_{\mathrm{g}}[\mathrm{kpc}]$ & - & 5 & 6 & 7 \\
\hline$h_{\mathrm{g}}[\mathrm{kpc}]$ & - & 0.2 & 0.2 & 0.2 \\
\hline
\end{tabular}
teristic radii $r_{\mathrm{B}}$ and $r_{\mathrm{H}} . M_{*}$ and $M_{\mathrm{g}}$ represent the masses of the stellar and gaseous disks, whose vertical and radial scale lengths are given, respectively, by $h_{*}$ and $a_{*}$, and $h_{\mathrm{g}}$ and $a_{\mathrm{g}}$.

Fig. 1. Hubble sequence for our galaxy models. From left to right, projection on the $x-z$ plane of a gE0, gSa, gSb and gSd galaxy. All the different components (gas, stars and dark matter) are plotted. Dark shading represents denser regions. Each frame is $20 \mathrm{kpc} \times 20 \mathrm{kpc}$ in size.

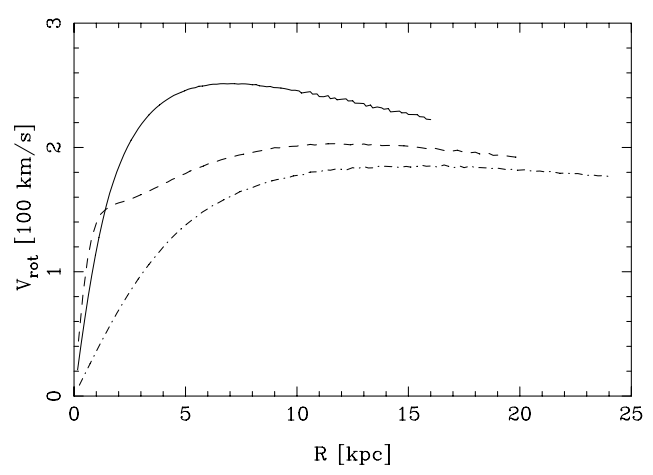

Fig. 2. Initial rotation curves for the gSa (solid line), gSb (dashed line) and gSd (dot-dashed line) galaxy.

Intending to obtain hundreds of simulations, each galaxy is made up of 120000 particles, distributed among gas, stars and dark matter, depending on the morphological type (see Table 2).

To initialize particle velocities, we adopted the method described in Hernquist (1993).

\subsection{Orbital parameters}

Seeking to exploit a vast range of orbital parameters, we performed 24 different simulations for each couple of interacting galaxies, varying the galaxies'orbital initial conditions, in order to have (for the ideal Keplerian orbit of two equal point masses of mass $m=2.3 \times 10^{11} M_{\odot}$ ) the first pericenter separation $r_{\text {per }}=$ 8,16 , and $24 \mathrm{kpc}$. For each of these separations, we varied the relative velocities at pericenter, in order to have one parabolic and three hyperbolic orbits of different energy. Finally, for each of the selected orbits, we changed the sign of the orbital angular momentum in order to study both direct and retrograde encounters. 
Table 2. Particle numbers for each galactic composant.

\begin{tabular}{ccccc}
\hline \hline & gE0 & gSa & gSb & gSd \\
\hline$N_{\text {gas }}$ & - & 20000 & 40000 & 60000 \\
$N_{\text {star }}$ & 80000 & 60000 & 40000 & 20000 \\
$N_{\text {DM }}$ & 40000 & 40000 & 40000 & 40000 \\
\hline
\end{tabular}

Table 3. Galaxies orbital parameters.

\begin{tabular}{cccccc}
\hline \hline Id & $\begin{array}{c}r_{\text {ini }} \\
{[\mathrm{kpc}]}\end{array}$ & $\begin{array}{c}r_{\mathrm{p}}{ }^{a} \\
{[\mathrm{kpc}]}\end{array}$ & $\begin{array}{c}\mathrm{v}_{\mathrm{p}}{ }^{a} \\
{\left[10^{2} \mathrm{~km} \mathrm{~s}^{-1}\right]}\end{array}$ & $\begin{array}{c}E^{a, b} \\
{\left[10^{4} \mathrm{~km}^{2} \mathrm{~s}^{-2}\right]}\end{array}$ & Spin $^{c}$ \\
\hline 01dir & 100. & 8.0 & 7.07 & 0.0 & up \\
01ret & 100. & 8.0 & 7.07 & 0.0 & down \\
02dir & 100. & 8.0 & 7.42 & 2.5 & up \\
02ret & 100. & 8.0 & 7.42 & 2.5 & down \\
03dir & 100. & 8.0 & 7.74 & 5.0 & up \\
03ret & 100. & 8.0 & 7.74 & 5.0 & down \\
04dir & 100. & 8.0 & 8.94 & 15.0 & up \\
04ret & 100. & 8.0 & 8.94 & 15.0 & down \\
05dir & 100. & 16.0 & 5.00 & 0.0 & up \\
05ret & 100. & 16.0 & 5.00 & 0.0 & down \\
06dir & 100. & 16.0 & 5.48 & 2.5 & up \\
06ret & 100. & 16.0 & 5.48 & 2.5 & down \\
07dir & 100. & 16.0 & 5.92 & 5.0 & up \\
07ret & 100. & 16.0 & 5.92 & 5.0 & down \\
08dir & 100. & 16.0 & 7.42 & 15.0 & up \\
08ret & 100. & 16.0 & 7.42 & 15.0 & down \\
09dir & 100. & 24.0 & 4.08 & 0.0 & up \\
09ret & 100. & 24.0 & 4.08 & 0.0 & down \\
10dir & 100. & 24.0 & 4.65 & 2.5 & up \\
10ret & 100. & 24.0 & 4.65 & 2.5 & down \\
11dir & 100. & 24.0 & 5.16 & 5.0 & up \\
11ret & 100. & 24.0 & 5.16 & 5.0 & down \\
12dir & 100. & 24.0 & 6.83 & 15.0 & up \\
12ret & 100. & 24.0 & 6.83 & 15.0 & down \\
\hline
\end{tabular}

${ }^{a}$ For two equal point masses of mass $m=2.3 \times 10^{11} M_{\odot}$.

${ }^{b}$ It is the total energy of the relative motion, i.e. $E=v^{2} / 2-G\left(m_{1}+m_{2}\right) / r$.

${ }^{c}$ Orbital spin, if parallel (up) or antiparallel (down) to the galaxies spin.

Combining each orbital configuration with all possible morphologies for the interacting pair of galaxies, we obtained a total sample of 240 interactions.

In Table 3, the initial distance $r_{\text {ini }}$ and the pericenter distance $r_{\mathrm{p}}$ between the galaxies center-of-mass are listed, together with their relative velocity $v_{\mathrm{p}}$ at pericenter and the orbital energy $E$, for all the simulated encounters ${ }^{2}$.

The results described in this paper refer only to planar encounters (galactic disks lying in the orbital plane).

\section{Numerical method}

To model galaxy evolution, we employed a Tree-SPH code, in which gravitational forces are calculated using a hierarchical tree method (Barnes \& Hut 1986) and gas evolution is followed by means of smoothed particle hydrodynamics (Lucy 1977; Gingold \& Monaghan 1982). Gravitational forces are calculated using a tolerance parameter $\theta=0.7$ and include terms up to the quadrupole order in the multiple expansion. A Plummer potential is used to soften gravitational forces, with constant softening lengths for different species of particles. In the simulations described here, if not explicitly stated otherwise, we assume $\epsilon=280 \mathrm{pc}$.

2 The values refer to the ideal Keplerian orbit of two equal point masses of mass $m=2.3 \times 10^{11} M_{\odot}$.
SPH (Smoothed particle hydrodynamics) is a Lagrangian technique in which the gas is partitioned into fluid elements represented by particles, which obey equations of motion similar to the collisionless component, but with additional terms describing pressure gradients, viscous forces and radiative effects in gas. To capture shocks a conventional form of the artificial viscosity is used, with parameters $\alpha=0.5$ and $\beta=1.0$ (Hernquist \& Katz 1989). To describe different spatial dynamical ranges SPH particles have individual smoothing lengths $h_{i}$, calculated in such a way that a constant number of neighbors lies within $2 h_{i}$. All the simulations have been performed using a number of neighbors $N_{\mathrm{s}} \sim 15$. The gas is modeled as isothermal, with a temperature $T_{\text {gas }}=10^{4} \mathrm{~K}$. Because of the short cooling time of disk gas, fluctuations in the gas temperature are quickly radiated away, so that simulations employing an isothermal equation of state differ little from more realistic ones (Mihos \& Hernquist 1996; Naab et al. 2006).

The equations of motion are integrated using a leapfrog algorithm with a fixed time step of $\Delta t=5 \times 10^{5} \mathrm{yr}$.

\subsection{Star Formation and continuous stellar mass loss}

Including star formation in gas dynamics is not a trivial task and a lot of different recipes and numerical methods can be adopted (Katz 1992; Steinmetz \& Müller 1994; Springel 2000; Springel \& Hernquist 2003; Cox et al. 2006), in order to model, on the one hand, the star formation rate and, on the other hand, to allow for the effects that this star formation has on the surroundings.

As in Mihos \& Hernquist (1994b), we parametrized the star formation efficiency for a SPH particle as

$\frac{\dot{M}_{\text {gas }}}{M_{\text {gas }}}=C \times \rho_{\text {gas }}{ }^{1 / 2}$

with the constant $C$ chosen such that the isolated disk galaxies form stars at an average rate of between 1 and $2.5 M_{\odot} \mathrm{yr}^{-1}$.

The choice of the parametrization in Eq. (5) is consistent with the observational evidence that on global scales the SFR in disk galaxies is well represented by a Schmidt law of the form $\Sigma_{\mathrm{SFR}}=A \Sigma_{\text {gas }}^{N}$, being $\Sigma_{\text {gas }}$ and $\Sigma_{\mathrm{SFR}}$ disk-averaged surface densities, with the best fitting slope $N$ about 1.4 (see Kennicutt 1998a; but also Wong \& Blitz 2002; Boissier et al. 2003; Gao \& Solomon 2004). Interestingly, this relation seems to apply, with a similar slope, also to local scales, as shown in Kennicutt et al. (2005) for M 51.

Once the SFR recipe is defined, we apply it to SPH particles, using the hybrid method described in Mihos \& Hernquist (1994b). This consists of representing each gas particle with two mass values, one corresponding to its gravitational mass $M_{i}$, whose value stays unchanged during the whole simulation, and the other describing the gas content of the particle $M_{i \text {,gas }}$, whose value changes over time, according to Eq. (5). Gravitational forces are always evaluated on the gravitational mass $M_{i}$, while hydrodynamical quantities, in turn, uses the time-varying $M_{i, \text { gas }}$. Only if the gas fraction present in the hybrid particles drops below $5 \%$ of the initial gas content, the hybrid particle is totally converted into a star-like particle and the small amount of gas material still present is spread over neighbors.

We also followed the method described in Mihos \& Hernquist (1994b) for including the effects of star formation into the ISM. The method is fully described in the paper cited above, and here we briefly recall only the main features. A MillerScalo stellar mass function was adopted, and we evaluated the fraction of stars with masses $>8 M_{\odot}$, assuming that they 
instantaneously become supernovae, leaving behind remnants of $1.4 M_{\odot}$ and releasing their mass to the surrounding ISM. The mass released also enriches the metallicity of the surrounding gas. This is done assuming a yield $y=M_{\text {ret }} / M_{*}=0.02, M_{\text {ret }}$ being the total mass of all reprocessed metals and $M_{*}$ the total mass in stars. For each gas particle, mass and metals return is applied to the $i-$ th neighbor gas particle, using a weight $w_{i}$ based on the smoothing kernel.

The energy injection in the ISM from SNe explosions is treated assuming that only a fraction $\epsilon_{\mathrm{kin}}$ of $E_{\mathrm{SN}}=10^{51} \mathrm{erg}$ goes into kinetic energy, by applying a radial kick to velocities of neighbor gas particles; thus, for each SNe explosion, the $i-$ th neighboring gas particle receives a velocity impulse directed radially away from the "donor", with a magnitude

$\Delta v_{i}=\left(\frac{2 w_{i} \epsilon_{\mathrm{kin}} E_{\mathrm{SN}}}{M_{i}}\right)^{1 / 2}$,

$w_{i}$ being, once again, the weighting based on the smoothing kernel and $M_{i}$ the mass of the receiver.

Evidently, this method has a certain number of free parameters, which clearly influence not only the star formation evolution, but, more generally, the global galaxy dynamics. Before moving on to perform simulations of galaxy encounters, we run a set of simulations of isolated galaxies, in order to check the dependency of the results on the gravitational smoothing length $\epsilon$, and on the fraction $\epsilon_{\text {kin }}$ of kinetic energy that is released, via SNe explosions, to the surrounding gas particles. For example, for the choice of the gravitational smoothing length, we performed 21 simulations of galaxies $\mathrm{gSa}, \mathrm{gSb}$ and $\mathrm{gSd}$, with $\epsilon=70,140,210,280,350,420$ and $490 \mathrm{pc}$, respectively. The results showed that too low a value for $\epsilon$ causes a conspicuous heating of the stellar disk, for all the morphological galactic types. An $\epsilon=280 \mathrm{pc}$, which corresponds (for gSa and $\mathrm{gSb}$ galaxies) to the average distance between stars in the disk seemed to represent a compromise between accuracy in the evaluation of gravitational forces and avoiding excessive relaxation effects. Also for the choice of the $\epsilon_{\mathrm{kin}}$ parameter, a set of numerical simulations of isolated galaxies was performed, and the results found were in good agreement to those of Mihos \& Hernquist (1994b), i.e. it was checked that if the total amount of kinetic energy received by a gas particle, due to the contribution from all its neighbors, is $\leq 1 \mathrm{~km} \mathrm{~s}^{-1}$, this prevents rapid growth of the vertical thickness of the gaseous disk, giving good results in terms of gas and star formation morphology.

If star formation acts in consuming the gas of a galaxy, an exhaustive modelling of galactic evolution must also take into account the competing process of stellar mass-loss. Indeed, as both observational studies and stellar evolutionary models propose, it can play an important role, since the gas mass fraction restituted by stars may reach some $45 \%$ over the Hubble time, when integrated over the stellar mass spectrum.

We included continuous stellar mass loss in the model adopting the formula given in Eq. (2) of Jungwiert et al. (2001), and applying it only to stellar populations formed into hybrid particles, i.e., at each time step, an amount

$M_{i, \mathrm{~s}}(t)=\frac{\left(M_{i}-M_{i, \mathrm{gas}}(t)\right) \Delta t c_{0}}{t-t_{\mathrm{birth}}+T_{0}}$

of the stellar mass in the population is lost by evolutionary effects, going to enrich the gas content $M_{i \text {,gas }}$ of the hybrid particle. In the formula above, $t_{\text {birth }}$ represents the birth time of the population, $T_{0}=4.97 \mathrm{Myr}$ and $c_{0}=5.47 \times 10^{-2}$ (see Jungwiert et al. 2001, for details).

\section{Results}

In this section, the main results of our study will be presented and discussed. This first paper being devoted to the study of star formation in interacting galaxy pairs, we will exclude from our analysis 24 simulations from the total sample of 240 , these simulations involving only gE0-gE0 encounters ("dry" interactions).

After presenting an image gallery of galaxy interactions and discussing the main morphological features arising during encounters (Sect. 4.1), we will move on to examine the evolution of star formation. To distinguish which contribution to the global SFR comes from the undisturbed galaxies and which from the interaction, we will first analyze the evolution of the star formation rate for the isolated galaxies (Sect. 4.2) and then describe the more salient features of the SFR evolution for the interacting pairs (Sect. 4.3). The evolution of the star formation efficiency (SFE) will also be discussed (Sect. 4.4), while in Sect. 4.5 we will describe where star formation regions are located.

The next step will consist in trying to understand which parameters determine the different SFR obtained soon after the pericenter passage and in the merging phase for different galaxy pairs. This will lead us to study in detail the dependency of the SFR on total gas content $M_{\text {gas }}$ in the galaxy just before the burst phase (Sect. 4.6), galaxy separation at pericenter passage $R_{\mathrm{p}}$, galaxies'relative velocity at pericenter passage $V_{\mathrm{p}}$, characteristic time of the encounter $t_{\mathrm{enc}}$, and tidal effects of the encounter, quantified by means of a suitable "tidal parameter" (Sect. 4.7). The dependency of the SFR on this tidal parameter will be gone into more deeply in Sect. 4.8, while in Sect. 4.9 we propose a formulation for the SFE in the burst phase. Section 4.10 presents a discussion of the evolution of interacting, merging and postmerger galaxies into the $\left(\Sigma_{\text {gas }}, \Sigma_{\mathrm{SFR}}\right)$ plane. Finally, in Sect. 4.11 gas inflow into the central galactic regions will be exploited.

In the following, we will often refer to the galaxy center in our analysis. If not otherwise written, we refer to a densityweighted center evaluated in the following way.

For each configuration, and for each galaxy:

- first we evaluate the density center (Casertano \& Hut 1985) of the dark matter particles $C_{\mathrm{DM}}$ initially $(t=0)$ belonging to the galaxy;

- then we evaluate the density center for all the particles (gas + stars + dark matter) initially $(t=0)$ belonging to the galaxy, that are at a distance $r<10 \mathrm{kpc}$ from $C_{\mathrm{DM}}$; the density center so found is used to define the center $C$ of the whole galaxy.

For evaluating the galaxy velocity, we evaluated the massweighted velocity of all the particles (stars + gas $+\mathrm{dm}$ ) at a distance $r<10 \mathrm{kpc}$ from $C$.

We will also refer sometimes to specific encounters. In this case the nomenclature adopted is the following: morphological type of the two galaxies in the interaction (gE0, gSa, gSb or $\mathrm{gSd}$ ), + the encounter identification string (see first column in Table 3), + 00 (for indicating a planar encounter). For example, the nomenclature gE0gSa04ret00 corresponds to a planar interaction between an elliptical and a Sa spiral, whose initial orbital parameters are those corresponding to id $=04$ ret in Table 3.

\subsection{A gallery of galaxy interactions}

Given the large number of simulations performed, it is not possible to describe each case individually. So we will proceed to describe some "fiducial" cases, that retain the mean features of 


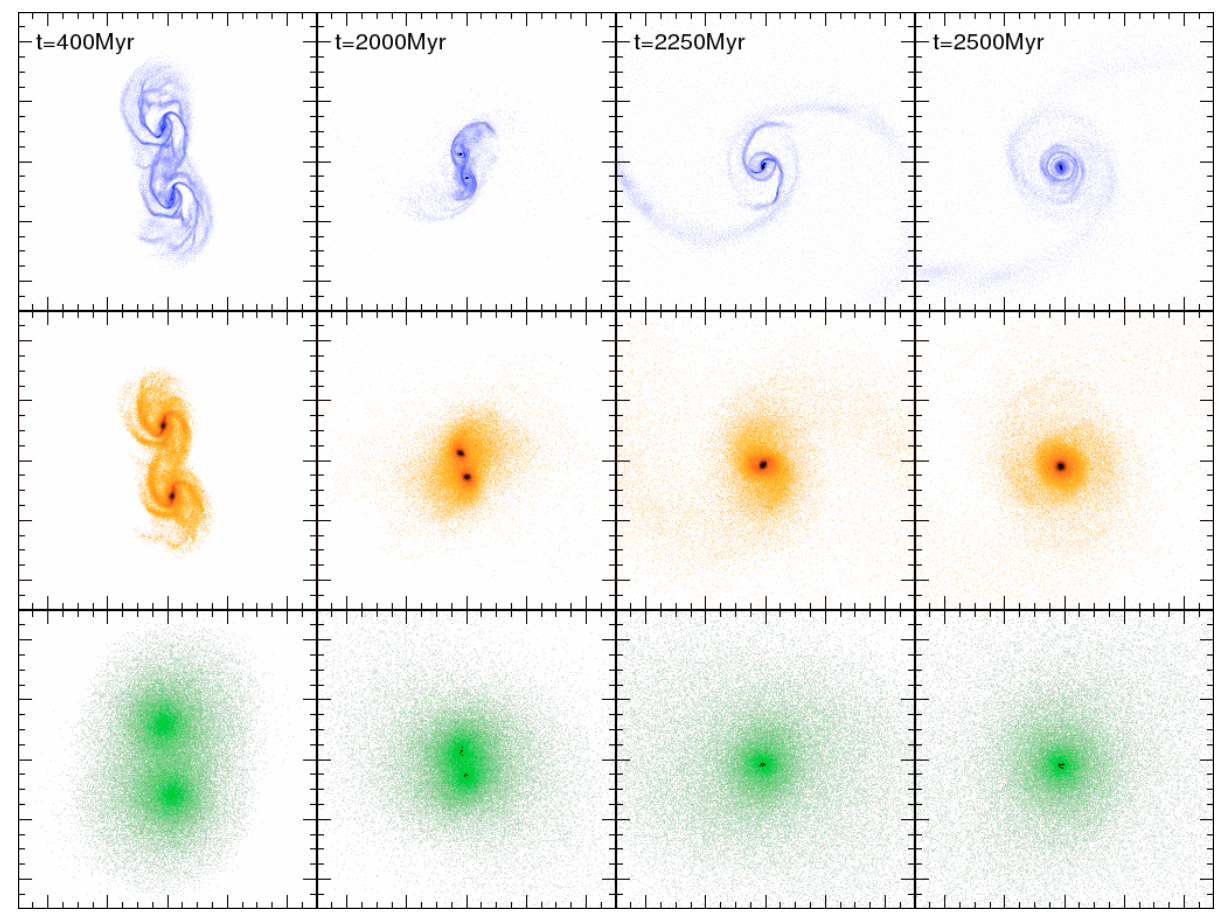

Fig. 3. Evolution of gas (upper panels), stars (intermediate panels) and dark matter (lower panels) during a direct merger between two gSb galaxies (id = 09dir in Table 3). Time is labelled in the upper part of the figure. Each frame is $50 \mathrm{kpc} \times 50 \mathrm{kpc}$ in size.

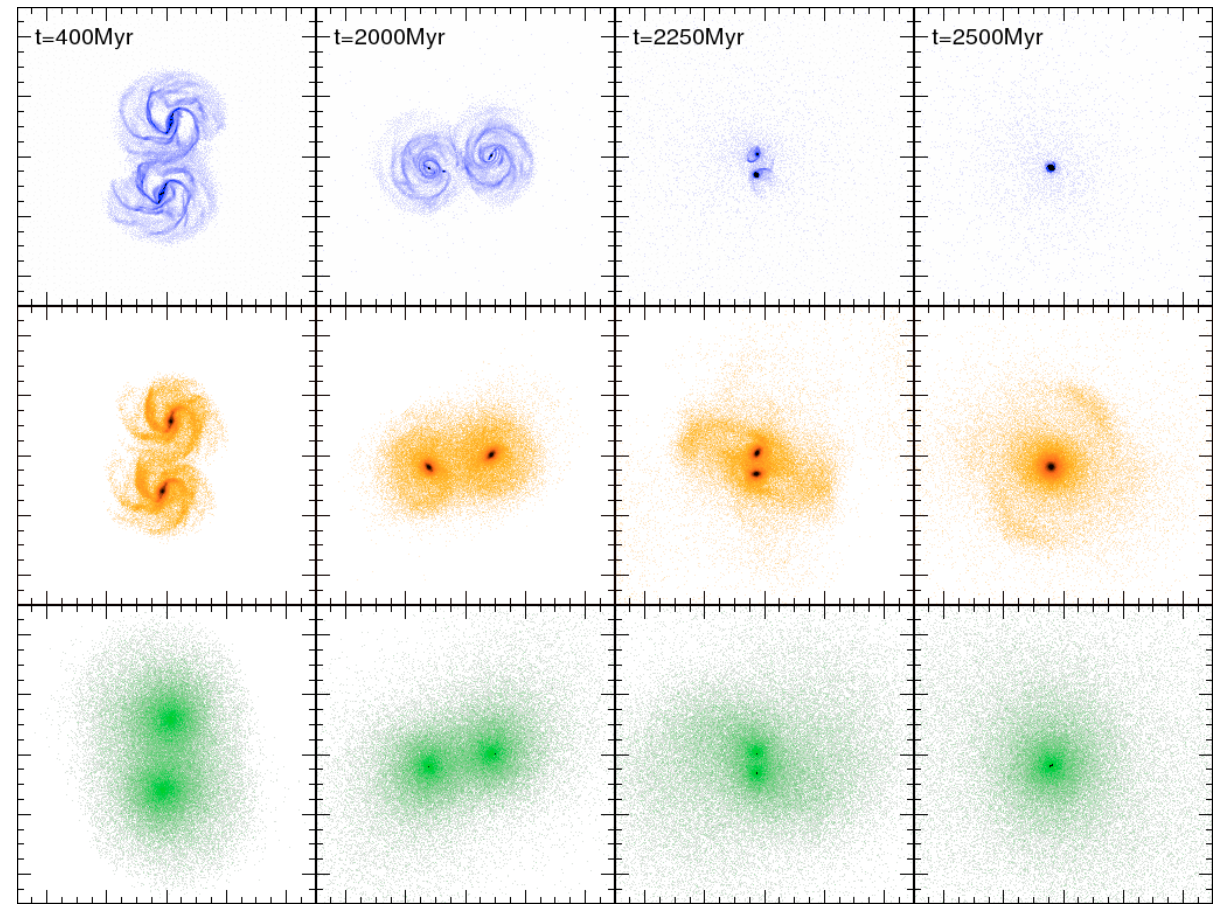

Fig. 4. Evolution of gas (upper panels), stars (intermediate panels) and dark matter (lower panels) during a retrograde merger between two gSb galaxies ( $\mathrm{id}=09 \mathrm{ret}$ in Table 3). Time is labelled in the upper part of the figure. Each frame is $50 \mathrm{kpc} \times 50 \mathrm{kpc}$ in size.

all the simulations performed. In Figs. 3-5, some sequences of galaxy mergings and flybys are shown.

Figure 3 describes the encounter and successive merger of two gSb galaxies, on direct orbit. At the beginning of the simulation the two galaxies are separated by a distance of $100 \mathrm{kpc}$. As they start to approach each other, they begin to develop tails, populated of both stars and gas particles. The intense tidal field exerted during the pericenter passage $(t=400 \mathrm{Myr})$ leads also to a transfer of mass between the two systems. In many cases, as it will be shown in the following sections, the direct encounter leads to a more rapid and dramatic merger, with an expansion of the outer parts of the system, which is particularly visible for disk galaxies.

The retrograde merger is shown in Fig. 4. In this case the two galaxies are less affected by the tidal interaction. The disk galaxies develop two transient great spiral arms, after the 


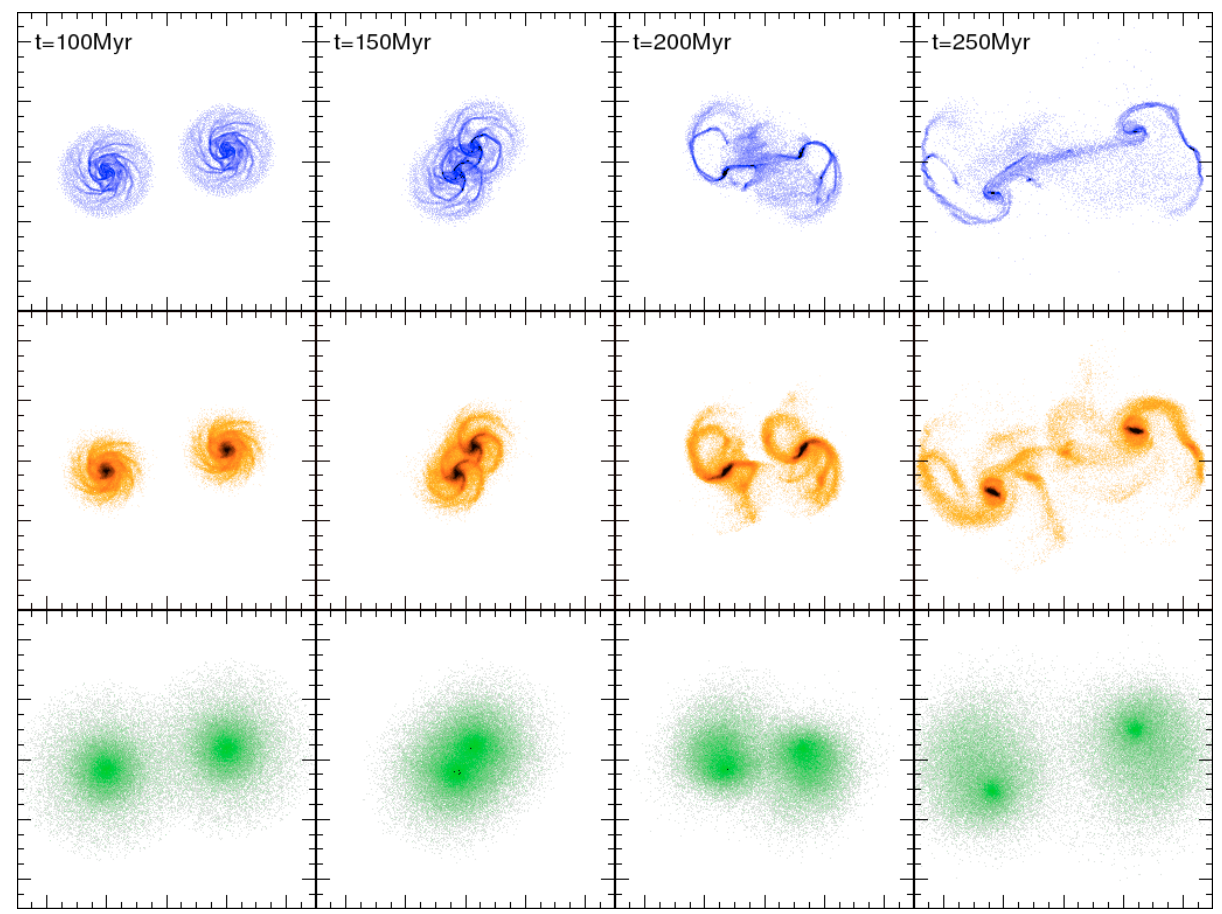

Fig. 5. Evolution of gas (upper panels), stars (intermediate panels) and dark matter (lower panels) during a direct flyby between two gSa galaxies (id =04dir in Table 3). Time is labelled in the upper part of the figure. Each frame is $50 \mathrm{kpc} \times 50 \mathrm{kpc}$ in size.

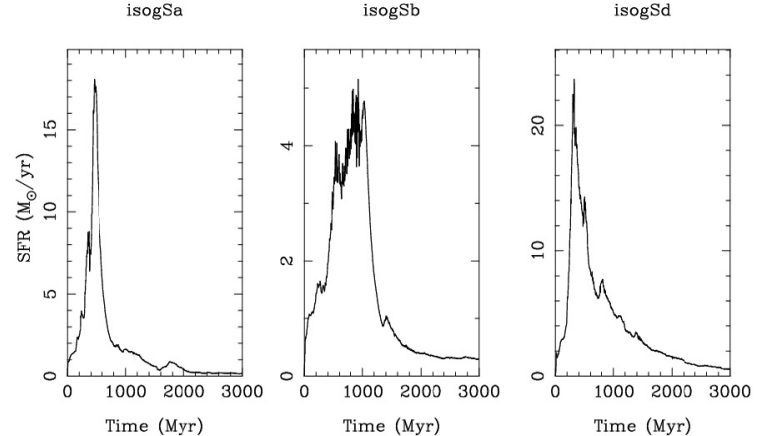

Fig. 6. From left to right: star formation rate of the isolated gSa, gSb and gSd galaxies.

pericenter passage, but retain their initial structure for much longer. Contrary to the direct case, no transfer of mass from one galaxy to the other takes place and the formation of tidal tails is less obvious. As will be discussed later, this allows most of the initial gas mass to stay well confined in the disk of the spiral galaxy, furnishing a great reservoir for the intense starburst that takes place in the final stage of the merging. Note particularly the high gas concentration in the inner central galactic regions in the last two snapshots of galaxies in Fig. 4.

Finally, Fig. 5 shows a direct flyby between two gSa spirals. Tidal tails and a bridge connecting the two galaxy centers develop after the pericenter passage at $t=170 \mathrm{Myr}$. In this, as in the previous figures, the high density regions in the gas component correspond also to the sites of most intense star formation, as it will be discussed in Sect. 4.5.

\subsection{Evolution of isolated galaxies}

Since the goal of this paper is to investigate the role interactions and mergers play in the star formation process, it is essential to

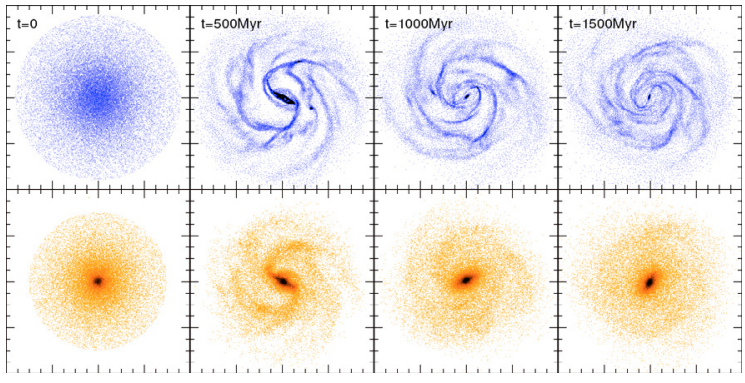

Fig. 7. Evolution of the gas component (upper panels) and of the stellar one (lower panels) for the isolated gSb galaxy. Time is labelled in the upper part of the figure. Each frame is $20 \mathrm{kpc} \times 20 \mathrm{kpc}$ in size.

study the evolution of the SFR in the isolated galaxies, in order to distinguish secular evolution from tidal effects in the interacting galaxy sample. This is shown in Fig. 6. In all three cases, an initial burst develops, driven by the compression of the gas into density waves. In Fig. 7, which shows the time evolution of the gaseous and stellar component for the gSb galaxy, spiral arms and a bar are clearly formed at $t=500 \mathrm{Myr}$.

After the initial peak, the SFR follows a typical exponential profile. This is a general result of simulations of galaxies evolved in isolation (see, for example, the discussions in Combes 2004; Harfst et al. 2006), unless ad hoc recipes for modeling feedback from SNe explosions are taken into account (Springel 2000). To reproduce the average constant star formation rates found in observations of spiral galaxies in the middle of the Hubble sequence (Kennicutt 1983; Kennicutt et al. 1994), it is indeed necessary to take into account also external gas accretion, as shown in simulations of galaxy evolution in a cosmological frame (Tissera 2000; Nagamine et al. 2004).

Averaged on the first $3 \mathrm{Gyr}$ of evolution, the $\langle S F R\rangle$ varies from $1 M_{\odot} /$ yr to $2.5 M_{\odot} /$ yr. 

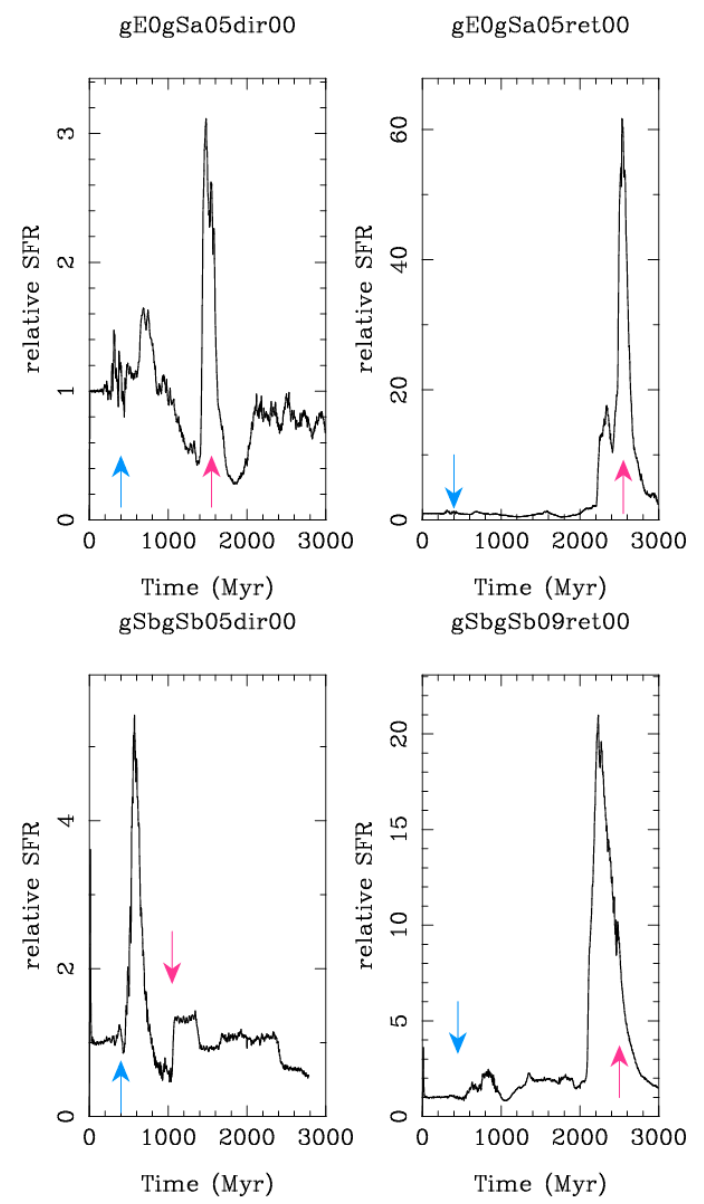

Fig. 8. Star formation rate, versus time, for some galaxy mergers. The SFR is normalized to that of the corresponding isolated galaxies. The blue arrows indicate the first pericenter passage between the two galaxies, and the red arrows the merger epoch.

\subsection{Star formation in interacting pairs}

The variety of morphological and orbital parameters adopted in the simulations is reflected in the different star formation histories of galaxies in the sample. Some SFR evolutions with time are shown in Figs. 8 and 9, which refer to mergings and flybys respectively. Simply looking at these few examples, it appears clear that interactions can increase star formation in the galaxy pairs from low levels (1.5-2 times the isolated case) to values typical of starburt galaxies (20-60 times the isolated case).

In these figures, no distinction is made between the two interacting galaxies in the sample, i.e. the relative SFR at time $t$ is evaluated as the ratio between the SFR of the pair and the total SFR of the isolated galaxies, both evaluated at time $t$. From Fig. 8, one can extrapolate some of the main features of the star formation evolution for interactions leading to mergers:

- the peak of the SFR usually occurs in the last phases of the encounter, when the two galaxies are interpenetrating, while, at the first pericenter passage, the increase in the SFR is only modest ( 1 to $4-5$ times that of the isolated case).

- for the same orbit, direct and retrograde encounters can lead to different SFR evolution. Obviously, this is not a general rule, but often the SFR is greater for retrograde mergers than direct ones and in some cases the differences between direct and retrograde encounters is conspicuous. For example in the $\mathrm{gE0gSa05ret00} \mathrm{encounter,} \mathrm{the} \mathrm{maximum} \mathrm{SFR} \mathrm{is} 20$ times
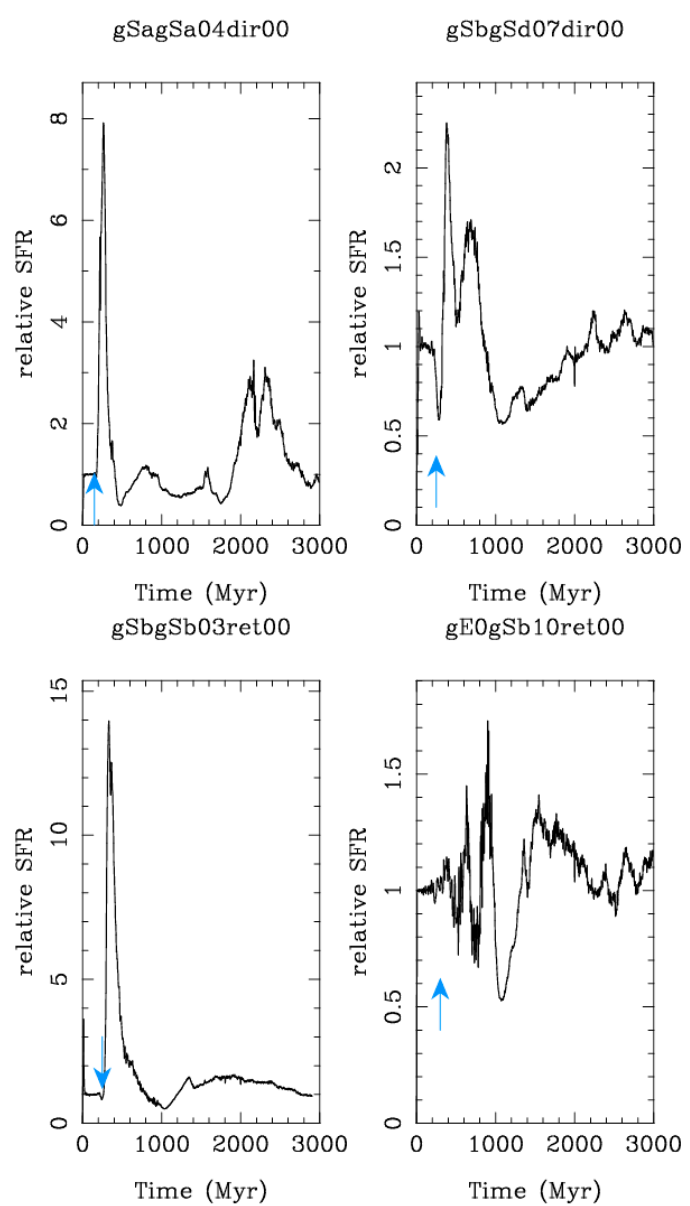

Fig. 9. Star formation rate, versus time, for some galaxy flybys. The SFR is normalized to that of the corresponding isolated galaxies. The blue arrows indicate the epoch of pericenter passage between the two galaxies.

greater than of the direct gE0gSa05dir00 encounter (upper panels in the figure).

For flybys, the peak in SFR occurs usually just after the pericenter passage; also in this case the increase in the star formation rate can be modest (as for the gSbgSd07dir00 and the gE0gSb10ret00 cases in Fig. 9) or substantial (cf. the gSagSa04dir00 and gSbgSb03ret00 in the same figure). On average, the SFR increases by a factor 3.4 with respect to the isolated galaxy sample.

Note that, both in Figs. 8 and 9, the peak in the star formation rate corresponds to the occurence of a nuclear starburst.

To show the wide variety of SFR obtained, in Fig. 10 we report the maximum SFR obtained during the 3 Gyr-evolution of the pairs, as a function of the different orbits simulated. As before, the SFR is relative to the isolated case. In this plot results are shown for the whole sample (i.e. both mergers and flybys). This clearly shows that, for the same initial orbital conditions, different morphologies of galaxies in the pair lead to different SFR histories, but also that, fixing the morphology of the pair, direct and retrograde encounters usually do not lead to the same star formation evolution. For mergers in general retrograde encounters are more efficient than direct ones in driving star formation: indeed, on average, in retrograde mergers the SFR peaks to 8.9 times that of the corresponding isolated galaxies, while, for direct mergers, the maximum star formation rate (always relative to the isolated case) is only 5 . 


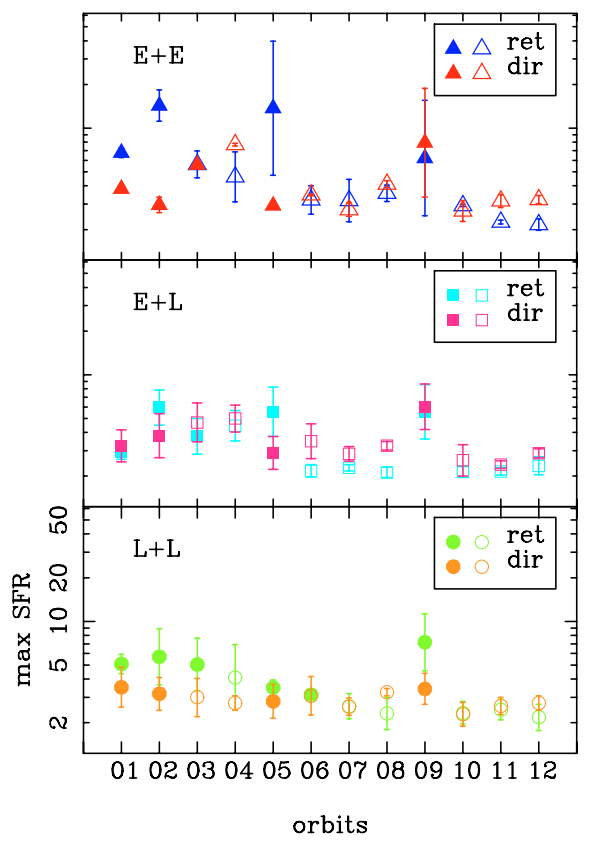

Fig. 10. Maximum star formation rate, relative to the isolated case, as a function of the orbital type, for flybys (empty symbols) and mergers (solid symbols). Encounters are grouped into three classes, depending on the morphology of the interacting galaxies: interactions between two early type galaxies are shown in the upper panel, the medium panel refers to early-late type encounters, the lowest to encounters involving two late-type systems. In each panel, error bars represent the standard error of the mean. The large error bars found in some cases reflect the great dispersion in the data.

\subsubsection{Integrated star formation}

Here we want to examine the evolution of the integrated star formation rate $\left(I S F R=\int_{t=0}^{t=3 \mathrm{Gyr}} S F R(t) \mathrm{d} t\right)$, i.e. the total quantity of gas transformed into stars, during the whole duration of the simulations. We have seen that different simulations show different SFRs; they are therefore more or less efficient, during pericenter passages or mergers, in transforming gas into stellar matter. It is interesting to check if the ISFR shows the same trend (for example if retrograde mergers are more efficient than direct ones), because, a priori, this is not guaranteed: ISFR can be dominated by an extreme starburst-like event for a short duration (several million years) or a long-term low enhancement of SFR due to interaction.

In Fig. 11, the ISFR, normalized to the isolated case, is shown, for all the simulations performed, as a function of the orbit. The main features are:

- Integrated on 3 Gyr of evolution, the total gas mass converted into stars can be increased by up to a factor of $\sim 2$, due to galaxy interactions.

- Flybys can be as efficient as mergers in transforming gas into stars: for example, two gSb galaxies, involved in a retrograde flyby, can have an ISFR comparable to that obtained in a merger (cf. the L-L flyby with orbit id $=03$ with the L-L mergers 05 and 09 in Fig. 11).

- In general, retrograde encounters are slighlty more efficient in transforming gas into stars: on average, for retrograde interactions $\langle I S F R\rangle=1.24$, while $\langle I S F R\rangle=1.15$ for direct encounters.

- The highest ISFR values are obtained for interactions involving late-type galaxies.

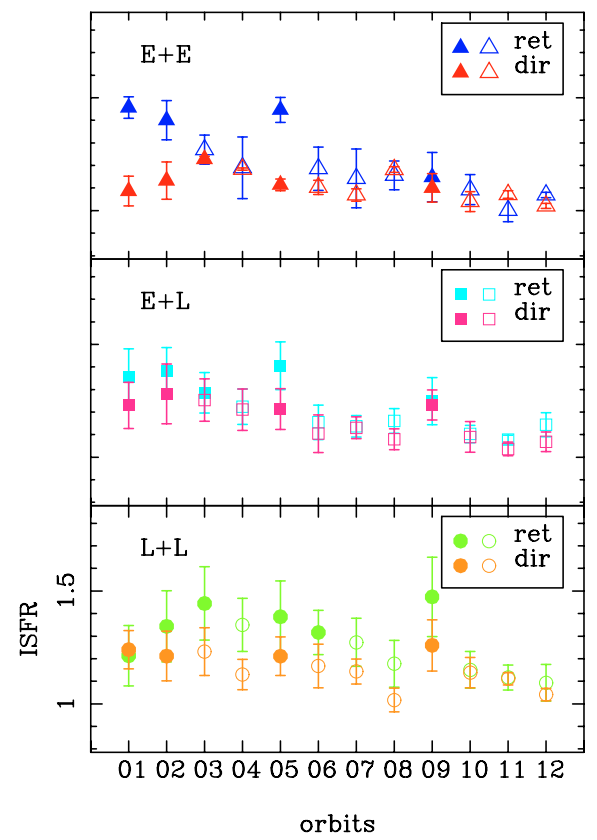

Fig. 11. Integrated star formation rate, ISFR, relative to the isolated case, as a function of the orbital type, for flybys (empty symbols) and mergers (solid symbols). Encounters are grouped into three classes, depending on the morphology of the interacting galaxies: interactions between two early type galaxies are shown in the upper panel, the medium panel refers to early-late type encounters, the lowest to encounters involving two late-type systems. In each panel, error bars represent the standard error of the mean. The large error bars found in some cases reflect the great dispersion in the data.

\subsection{Star formation efficiency in interacting pairs}

To describe how much stars are produced by unit gas mass, it is useful to study the star formation efficiency (SFE). This parameter can be defined as the ratio between the amount of gas transformed into stars, at a generic time $t$, and the available gas content at the same time:

$\operatorname{SFE}(t)=\frac{M_{\mathrm{gas} \rightarrow \star}(t)}{M_{\mathrm{gas}}(t)}$

Several authors have pointed out that strongly interacting galaxies are also more efficient in forming stars (Solomon \& Sage 1988; Combes et al. 1994; Georgakakis et al. 2000), while others (Casasola et al. 2004) have found that, even if interacting galaxies appear more luminous in the infrared, this higher star formation rate does not correspond to a different efficiency in star formation per unit of calculated $\mathrm{H}_{2}$ mass.

In Fig. 12 the SFE, relative to that of the corresponding isolated galaxies, is shown for some of the simulations performed. In all the cases, we found that an increase in the SFR is correlated with an increase in the SFE, as expected from Eq. (8). It is interesting to note, however, that, for the most intense bursts:

- usually the SFE peak is higher than the corresponding SFR and is also slightly delayed in time;

- the characteristic SFE times are greater than the corresponding SFR ones, i.e., after the encounter/merger many galaxies can show a SFR which is returned at preinteraction levels, while the SFE still shows higher values in comparison to the isolated counterparts.

This is evidently a consequence of the adopted SFE definition, because of the fact that, during an intense burst of star formation, 

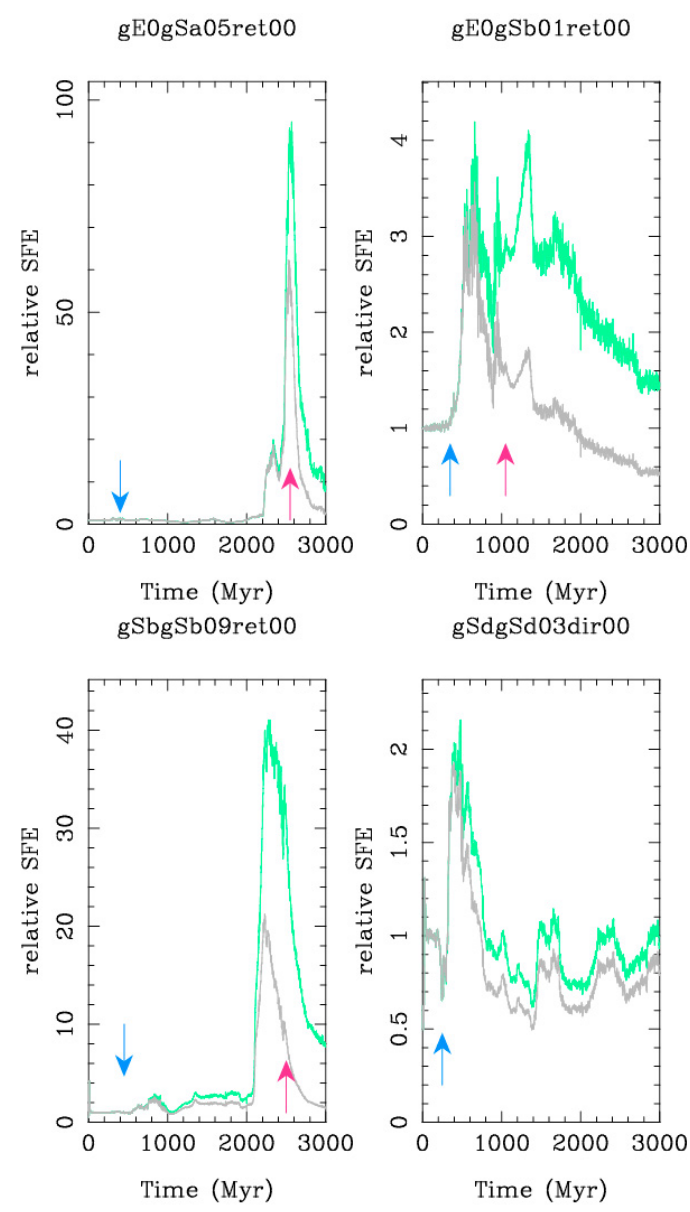

Fig. 12. Star formation efficiency (cyan curve), relative to the isolated case, as a function of time for three mergers (gE0gSa05ret00, gE0gSb01ret00, gSbgSb09ret00) and a flyby (gSdgSd03dir00). The blue arrows indicate the first pericenter passage between the two galaxies, and the red arrows the merger epoch. For comparison also the relative SFR is shown (grey curve).

gas in interacting galaxies is more rapidly depleted, with respect to the isolated case, thus the total amount of gas content in the galaxies is then lower than that of the isolated counterpart.

In Fig. 13, the maximum star formation efficiency obtained for each encounter is shown, as a function of the orbit.

\subsection{Where do stars form?}

From the results presented in the previous sections it is clear that interactions and mergers can lead to a conspicuous enhancement both in the SFR and in SFE (up to a factor of 100 for the SFE relative to the isolated case), but this is not always so. The picture that begins to emerge is that interactions, and mergers in particular, can lead to intense starbursts, but this is not always the case, i.e. galaxy interactions are not a sufficient condition to convert high gas mass quantities into new stars (see Bergvall et al. 2003 for an observational study of a sample of interacting galaxies that result in poor starburst triggers).

While, up to now, numerical simulations seem to support mainly the idea of a frequent occurence of tidally triggered central starbursts (Mihos \& Hernquist 1994a,b, 1996; Springel 2000), observational results depict a more complex scenario. Ultra Luminous Infrared Galaxies (ULIRGs) indicate centrally concentrated star formation (but see also Combes et al. 2006 for

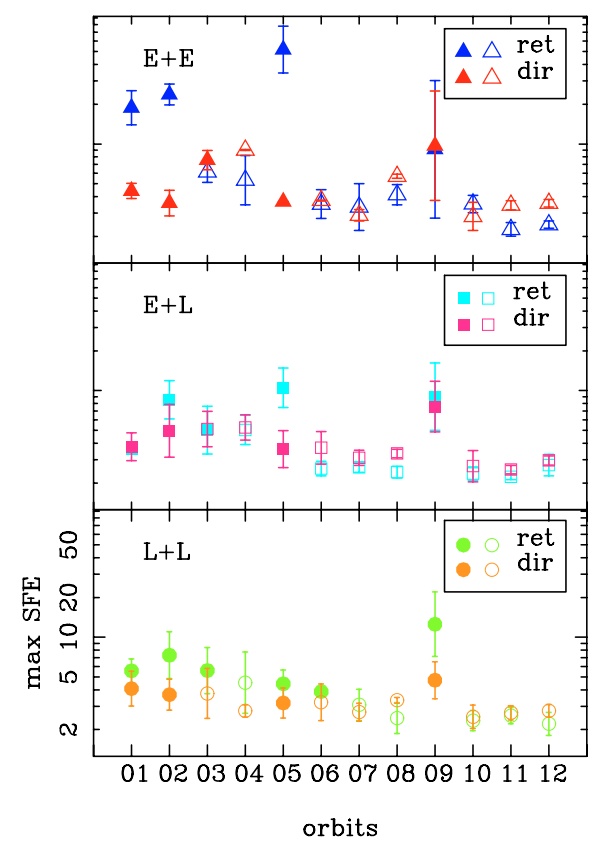

Fig. 13. Maximum star formation efficiency, relative to the isolated case, as a function of the orbital type, for flybys (empty symbols) and mergers (solid symbols). Encounters are grouped into three classes, depending on the morphology of the interacting galaxies: interactions between two early type galaxies are shown in the upper panel, the medium panel refers to early-late type encounters, the lowest to encounters involving two late-type systems. In each panel, error bars represent the standard error of the mean. The large error bars found in some cases reflect the great dispersion in the data.

an example of a moderate redshift ULIRG with a more extended CO emission), while a certain number of interacting galaxies feature a more extended star formation. In the Antennae galaxies (Wang et al. 2004), for example, the most intense star formation regions are located between the two galaxies, and the late-type galaxy NGC 275 in the Arp 140 system, despite being close to a merger with its companion NGC 274, does not show evidence of an enhanced or centrally concentrated star formation, the brightest emission from star formation tracers coming from an off-center region (Cullen et al. 2006).

Figures 14-19 show a comparison of most intense star formation regions and gas maps for three mergers of the sample. Far from representing the whole sample, they clearly suggest that the tidally triggered star formation process can manifest itself in a variety of ways. The retrograde encounter/merger between two late type $\mathrm{Sb}$ galaxies leads to a strong enhancement in the total $^{3}$ SFR (about 20 times that of the isolated case), the site of most intense star formation being strongly centrally concentrated. The same encounter, this time involving galaxies in direct orbits, leads to the star formation maps shown in Fig. 16: in this case the central galaxy regions are sites of intense star formation in the final phases of the merging process, but, this time, the tidal tails and the bridge connecting the two galaxies contribute also to the production of new stars, as well as circumnuclear rings ${ }^{4}$. The situation changes drastically in Fig. 18, where the star formation maps resulting from an encounter between an early-type elliptical and a late-type Sd galaxy are shown. In this case, the

\footnotetext{
3 i.e. evaluated on the total gas mass of the galaxy.

4 Note that the presence of an ILR stops the gas inflow. This, together with the tidal gas removal, explains why SFRs tend to be lower in direct orbits, as we will see later on.
} 


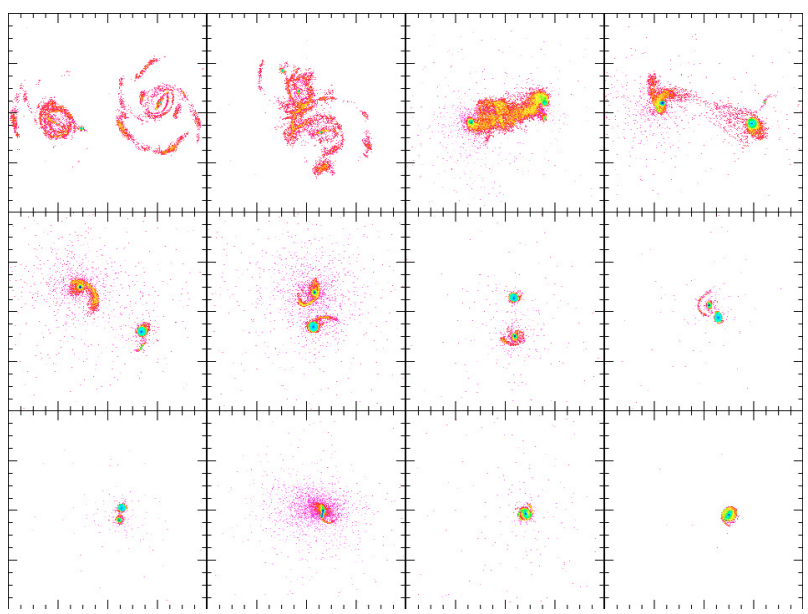

Fig. 14. Star forming regions during the final stage of an encounter involving two gSb galaxies in retrograde orbits $(\mathrm{id}=09$ ret in Table 3 ). Only hybrid particles with a star formation efficiency (averaged on $50 \mathrm{Myr}$ ) greater than 0.005 are shown. Each frame is $20 \mathrm{kpc}$ in length. Snapshots are shown every $50 \mathrm{Myr}$. Blue-green colors correspond to regions of highest density. See Fig. 15 for a comparison with gas maps.

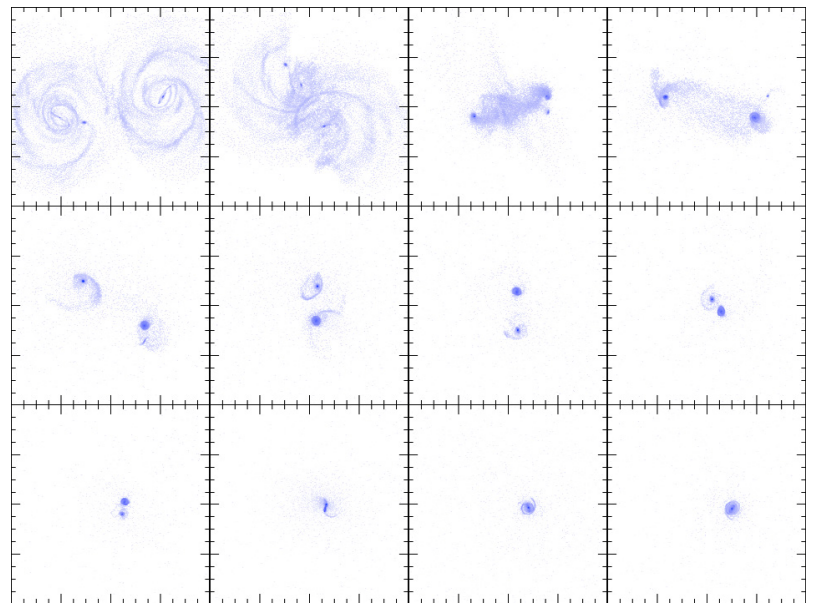

Fig. 15. Gas maps during the final stage of an encounter involving two gSb galaxies in retrograde orbits (id $=09$ ret in Table 3 ). Each frame is $20 \mathrm{kpc}$ in length. Snapshots are shown every 50 Myr. See Fig. 14 for a comparison with star forming regions.

first pericenter passage between the two galaxies is highly disruptive for the $\mathrm{gSd}$, which develops two giant tails, which acquire a high fraction of gas mass, and which are also the site of intense, local, and clumpy star formation. Note that the star forming clump in the upper left part of the panels in Fig. 18 corresponds to the center of the elliptical galaxy, which has acquired part of the gas mass of the companion during the interaction.

On average, the gas mass being spread at great distances from the galaxy center, the gas density is obviously lower than the one obtained in the above-mentioned encounter. This leads to a total SFR that is noticeably lower than the one obtained in the other two mergers: it is only three times greater than in the isolated case.

\subsection{How does the maximum SFR depend on the total amount of gas available in the galaxies?}

Before moving on to describe in detail the dynamical evolution of gas and stars during the encounters, in order to try to

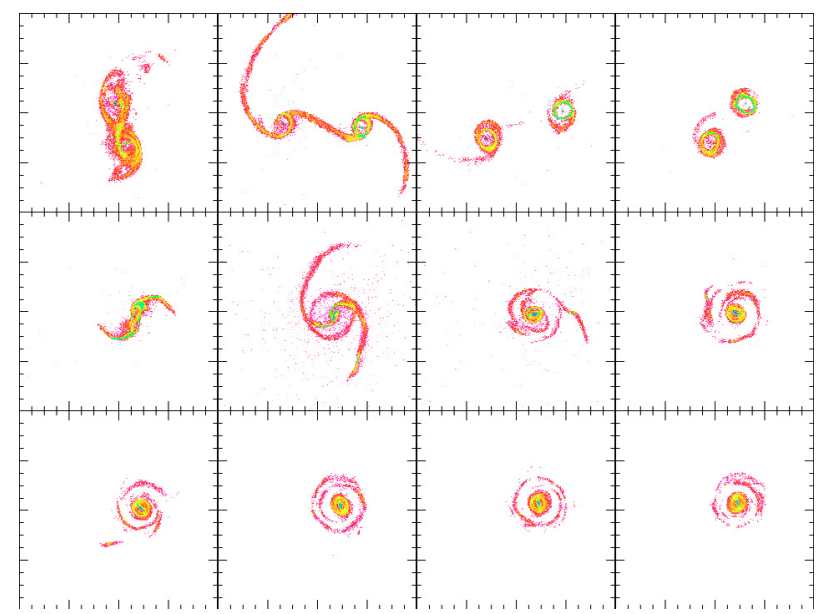

Fig. 16. Star forming regions during the final stage of an encounter involving two gSb galaxies in direct orbits (id $=09$ dir in Table 3$)$. Only hybrid particles with a star formation efficiency (averaged on $50 \mathrm{Myr}$ ) greater than 0.005 are shown. Each frame is $20 \mathrm{kpc}$ in length. Snapshots are shown every $50 \mathrm{Myr}$. Blue-green colors correspond to the regions of highest density. See Fig. 17 for a comparison with gas maps.

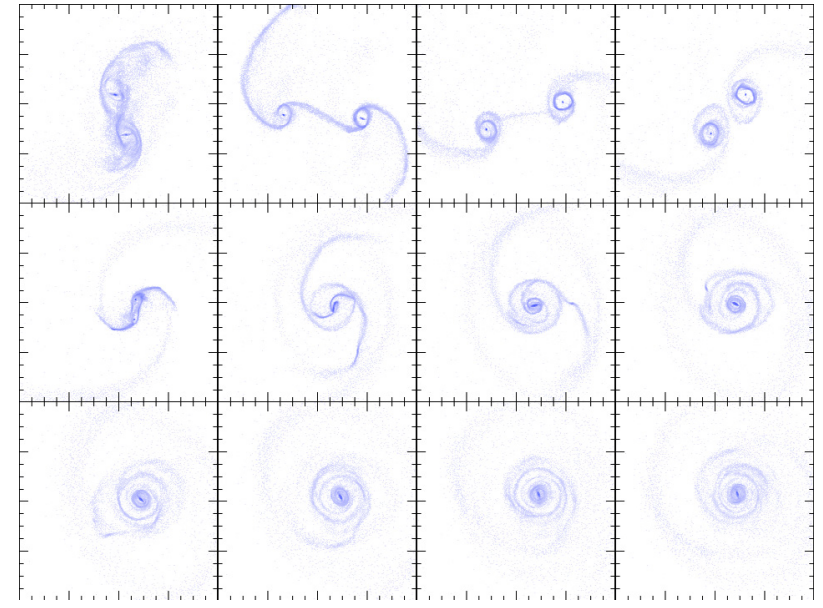

Fig. 17. Gas maps during the final stage of an encounter involving two gSb galaxies in direct orbits (id $=09$ dir in Table 3). Each frame is $20 \mathrm{kpc}$ in length. Snapshots are shown every 50 Myr. See Fig. 16 for a comparison with star forming regions.

understand the physical processes that lead to an enhancement in the SFR and in the ISFR, with respect to the isolated cases, we want to find out whether, and to what extent, the SFR peaks depend on the amount of gas available in the galaxy. In other words, are the different SFR peak values shown in Figs. 8-12 due to a different gas content in the galaxy at the moment of the burst?

To investigate this, we evaluated the gas content ${ }^{5}$ (relative to that at the beginning of the simulation) just before the burst, i.e. for flybys we evaluated the amount of gas in the galaxy $50 \mathrm{Myr}$ before the pericenter passage, while, for mergers, the quantity is evaluated $50 \mathrm{Myr}$ before the coalescence between the two galaxies. In Fig. 20 the maximum SFR is plotted, as a function of the gas content, expressed in units of the initial galactic gas mass, for all the encounters. Flybys and mergers are located in two different regions of the plots. For flybys, the pericenter passage occurs

5 The gas content considered is the total amount of gas, i.e. the gas can be present both in the tails or in the main body of the galaxy. 


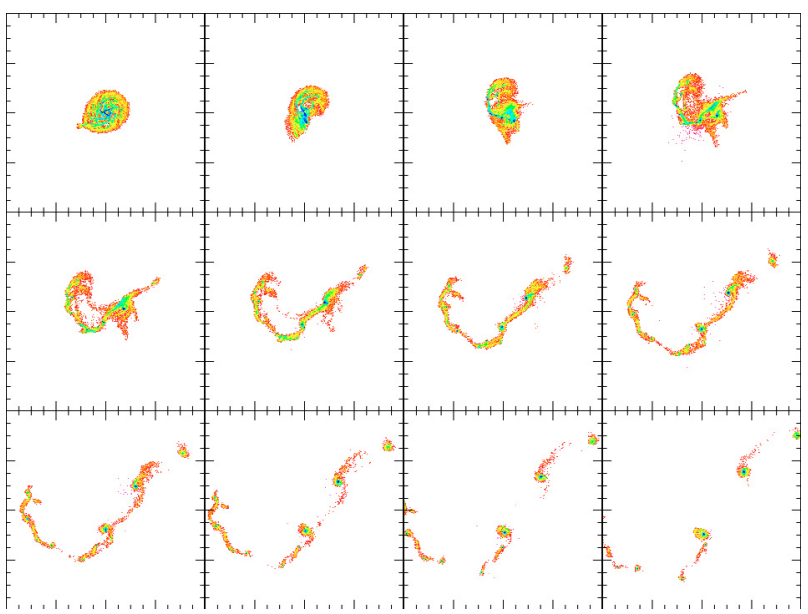

Fig. 18. Star forming regions during the final stage of an encounter involving a gE0 and a gSd galaxy in direct orbits (id $=03$ dir in Table 3 ). Only hybrid particles with a star formation efficiency (averaged on $50 \mathrm{Myr}$ ) greater than 0.005 are shown. Each frame is $100 \mathrm{kpc}$ in length. Snapshots are shown every $50 \mathrm{Myr}$. Blue-green colors correspond to the regions of highest density. See Fig. 19 for a comparison with gas maps.

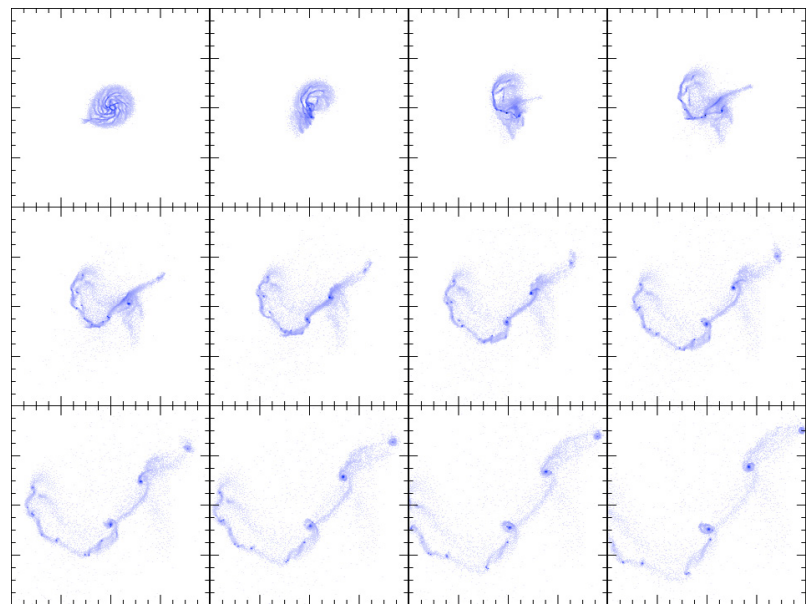

Fig. 19. Gas maps during the final stage of an encounter involving a gE0 and a gSd galaxy in direct orbits (id = 03dir in Table 3). Each frame is $100 \mathrm{kpc}$ in length. Snapshots are shown every $50 \mathrm{Myr}$. See Fig. 18 for a comparison with star forming regions.

in the initial phases of evolution, when the gas content of the disk galaxies is similar to the initial ones; mergers, on the other hand, are located in a region of space where the amount of gas mass is a fraction of between 0.2 and 0.4 of the initial gas mass for elliptical-spiral encounters, while it is somewhat greater range (0.1-0.6) for spiral-spiral ones.

In both cases (flybys and mergers) it is evident that one can have large differences in the maximum SFR, even when the amount of fuel available is nearly the same: this is the case of flybys involving a gE0 and a gSb in direct orbits, for example, where one can have a factor of 4 in the maximum SFRs, even if the gas mass is comparable. Even more striking is the case of mergers where, with an available gas mass which is about $33 \%$ of the initial value, there are encounters which lead to a maximum SFR 60 times the isolated case, while others enhance the SFR only by a factor of 5 .

In conclusion, Fig. 20 clearly shows that the quantity of fuel still available in the galaxy is not the main parameter that influences the SFR in the burst phase.

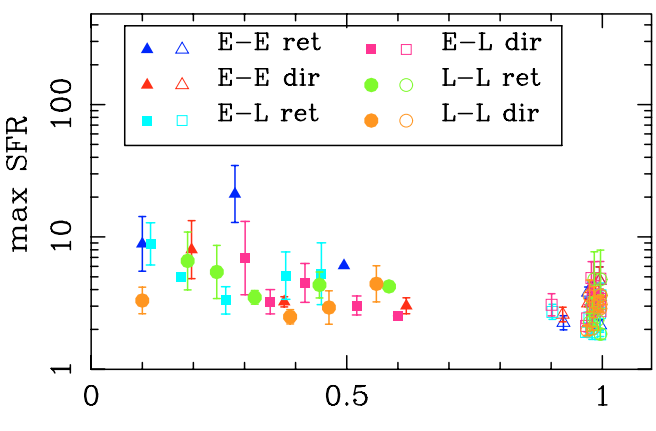

Gas Mass

Fig. 20. Maximum star formation rate (relative to the isolated case), as a function of the gas content (rescaled to its initial value), for flybys (empty symbols) and mergers (solid symbols). Different symbols correspond to different morphologies of interacting galaxies, as explained in the legend. Error bars represent the standard error of the mean.

\subsection{How does the maximum SFR depend on $R_{p}, V_{p}, t_{e n c}$ and on the effect of tidal forces?}

In the previous section we showed that the maximum SFR in interacting and merging galaxies does not depend mainly on the total amount of gas mass available in the main body of the system and in the tails. A first indication of the fact that the encounter geometry affects the enhancement of star formation has been seen in Sect. 4.3, comparing retrograde and direct encounters: we have seen that usually retrograde interactions/mergers are more efficient than direct ones in converting gas to stars. But it is still clear that other parameters must play a role in the evolution of the SFR in interacting galaxies: even considering only retrograde encounters between galaxies with the same morphology, in some cases the increase in the SFR relative to the isolated evolution is only modest, while other events lead to a SFR 60 times greater than that of the isolated case.

So, the aim of this section is to explore the dependency of the maximum SFR (occurring in the coalescence phase for mergers and soon after the pericenter passage for flybys) on other parameters, such as:

- the distance at the first pericenter passage $R_{\mathrm{p}}$;

- the relative velocity $V_{\mathrm{p}}$ at $R_{\mathrm{p}}{ }^{6}$;

- the characteristic time of the encounter, $t_{\mathrm{enc}}=\frac{R_{\mathrm{p}}}{V_{\mathrm{p}}}$

- the tidal parameter $T_{\mathrm{p}}$ of the galaxy pair, defined as ${ }^{7}$

$T_{\mathrm{p}}=T_{\mathrm{p}, 1}+T_{\mathrm{p}, 2}$,

where

$$
T_{\mathrm{p}, i}=\log _{10}\left[\frac{M_{\text {comp }}}{M_{i}}\left(\frac{D_{i}}{R_{\mathrm{p}}}\right)^{3}\right], i=1,2
$$

quantifies the effect of tidal forces at pericenter passage $R_{\mathrm{p}}$, suffered by a galaxy of mass $M_{i}$ and scalelength $D_{i}$, due to the interactions with a companion galaxy whose mass is $M_{\text {comp }}$ (see also Bournaud et al. 2005). As a measure of the

\footnotetext{
${ }^{6} R_{\mathrm{p}}$ and $V_{\mathrm{p}}$ have been evaluated using the definitions of galaxy centers and velocities given at the beginning of Sect. 4 .

7 Note that in the following definition of $T_{\mathrm{p}}$ no dependency on the galaxy mass ratio is proposed. The sample being composed only by equal mass galaxies, this has no influence on the present study. A possible formulation for unequal mass encounters could consist into introducing a weight $g=M_{i} /\left(M_{i}+M_{\text {comp }}\right)$ in the expression for $T_{\mathrm{p}, i}$, i.e. $T_{\mathrm{p}, i}=\log _{10}\left[g\left(M_{\text {comp }} / M_{i}\right) \times\left(D_{i} / R_{\mathrm{p}}\right)^{3}\right]$.
} 
Mergers

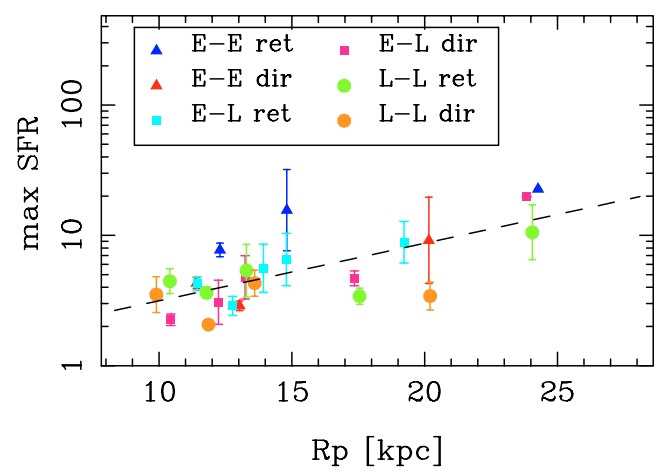

Flybys

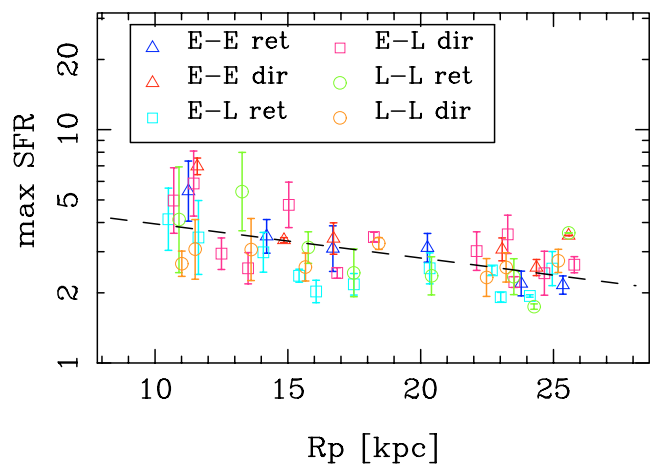

Fig. 21. Maximum star formation rate, relative to the isolated case, as a function of the first pericenter distance between the two galaxies for flybys (lower panel) and mergers (upper panel). Different symbols correspond to different morphologies of interacting galaxies, as explained in the legends. The dashed lines represent the best linear least-square fits. Error bars represent the standard error of the mean.

galaxy scalelength $D_{i}$ we used the radius containing $75 \%$ of the total (baryonic + dark matter) mass of the system;

- the parameter $T_{\mathrm{p}} / t_{\mathrm{enc}}$ that gives an estimate of the effect of tidal forces exterted on the pair, per unit of time.

We wish to remind readers that, while for flybys this analysis relates the SFR at pericenter passage with quantities evaluated at the pericenter too, this is not the case for mergers, looking in this case for possible existing correlations between the SFR in the coalescence phase and physical quantities evaluated at the first pericenter passage.

\subsubsection{Maximum SFR versus $R_{p}$}

Figure 21 shows the maximum SFR, as a function of the distance $R_{\mathrm{p}}$ at first pericenter passage, for flybys (lower panel) and mergers (upper one).

It is not surprising to see that for flybys there is a tendency to have increasing star formation levels as the pericenter separation of galaxies in the pair diminishes. More interesting is the case of mergers, where a positive correlation $(+0.7)$ between these two quantities is found, so that galaxies that at first passage are more distant are also those that suffer the most intense burst of star formation in the merging phase.
Mergers

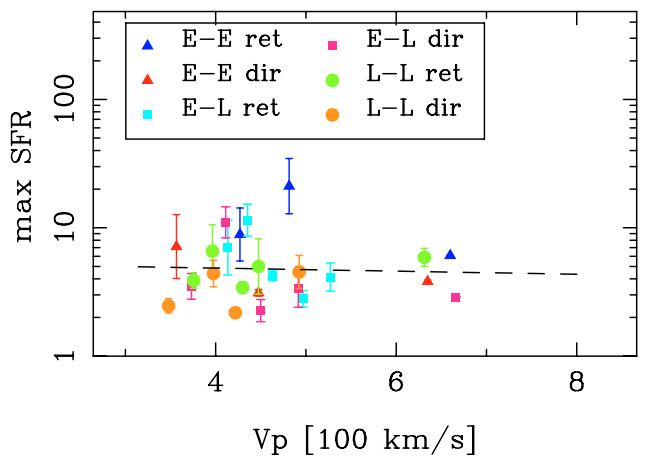

Flybys

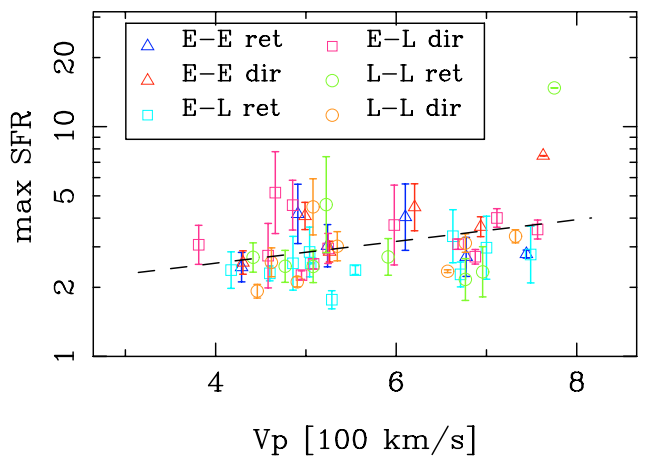

Fig. 22. Maximum star formation rate, relative to the isolated case, as a function of the relative velocity at pericenter, for flybys (lower panel) and mergers (upper panel). Different symbols correspond to different morphologies of interacting galaxies, as explained in the legends. The dashed lines represent the best linear least-square fits. Error bars represent the standard error of the mean.

\subsubsection{Maximum SFR versus $V_{p}$}

No correlation is found between SFR maximum and the relative velocity of the two galaxies at the first pericenter passage for the merger sample, while a weak correlation $(+0.34)$ exists for flybys (see Fig. 22).

\subsubsection{Maximum SFR versus $t_{\text {enc }}$}

As shown in Fig. 23, the duration of the encounter, and thus the duration of the perturbation induced by the companion galaxy, is a parameter that influences the peak of star formation (the absolute value of the correlation coefficient is about 0.6 for both flybys and mergers). And, as previously found for $R_{\mathrm{p}}$, the two samples of flybys and merger galaxies feature quite different behavior: indeed, while the maximum SFR increases as $t_{\text {enc }}$ diminishes for flybys, the opposite is the case for mergers, having a tendence to have higher bursts of star formation at increasing $t_{\text {enc }}$. This is exactly the same trend shown in Fig. 21, because of the fact that flybys with small pericentric distances must also have the highest relative velocities, and so, ultimately, small $t_{\text {enc }}$.

\subsubsection{Maximum SFR versus $T_{p}$}

For mergers a strong negative correlation $(-0.72)$ is found when plotting the maximum SFR versus the tidal parameter $T_{\mathrm{p}}$, defined as the sum of the tidal parameters $T_{\mathrm{p}}$ exerted by the 
Mergers

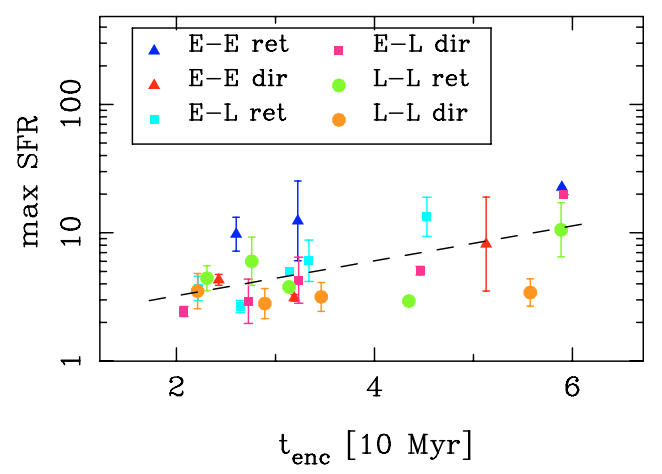

Flybys

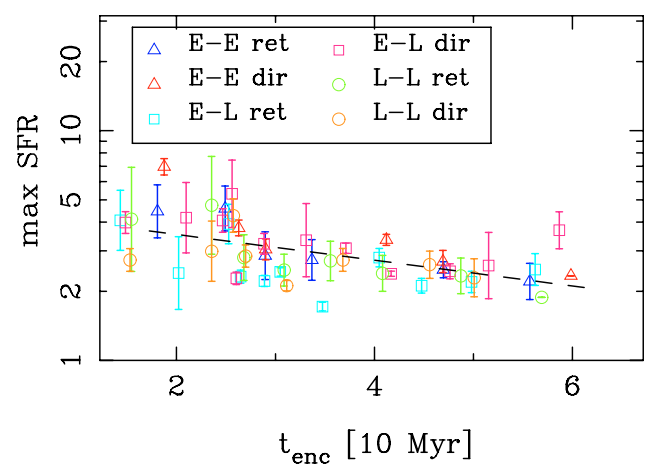

Fig. 23. Maximum star formation rate, relative to the isolated case, as a function of the characteristic time of the encounter $t_{\text {enc }}$, for flybys (lower panel) and mergers (upper panel). Different symbols correspond to different morphologies of interacting galaxies, as explained in the legends. The dashed lines represent the best linear least-square fits. Error bars represent the standard error of the mean.

two galaxies. The clear trend in Fig. 24 is that galaxy pairs that suffer intense tidal effects at first pericenter passage tend to have lower star formation rates in the merging phase. The same behavior is found when comparing the SFE at the merger epoch with $T_{\mathrm{p}}$ (see Fig. 25). The opposite trend stands for flybys, i.e. at pericenter, galaxy pairs that suffers intense tidal effects tend to have also higher star formation rates.

\subsubsection{Maximum SFR versus $T_{p} / t_{\text {enc }}$}

The same trend is found when comparing the SFR at the merger with $T_{\mathrm{p}} / t_{\mathrm{enc}}$, which gives an estimate of the perturbation due to tidal forces per unit time. With a correlation coefficient $r=$ -0.55 , it shows that the maximum SFR is somewhat affected by the tidal forces exerted by the companion galaxy per unit time. Remember that our star formation law is only density dependent, so that an increase in the SFR corresponds to an increase in the local gas density. In this sense, the negative correlation found between the amplitude of the burst and the tidal forces exerted per unit of time can be understood if one considers that the stronger the forces, the greater the quantity of galaxy mass (and so, ultimately, also of gas material) removed from the system, as an effect of energy injection (see the next section for a more in-depth discussion of this).
Mergers

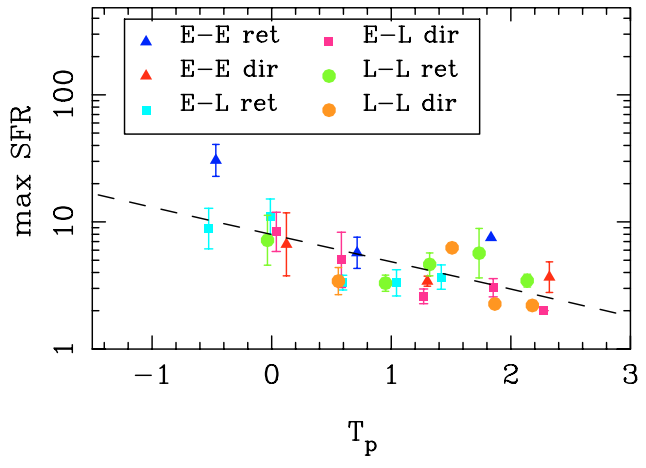

Flybys

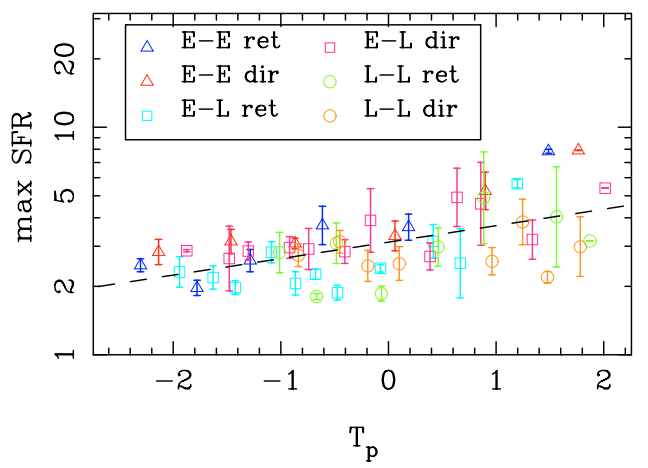

Fig. 24. Maximum star formation rate, relative to the isolated case, as a function of the tidal parameter $T_{\mathrm{p}}$ (see text), for flybys (lower panel) and mergers (upper panel). Different symbols correspond to different morphologies of interacting galaxies, as explained in the legends. The dashed lines represent the best linear least-square fits. Error bars represent the standard error of the mean.

\subsection{Why does the SFR in the merging phase depend on $T_{p}$ ?}

The goal of this section is to further investigate the relation found in Fig. 24 between the value of the SFR peak in the merging phase and effects of tidal forces on the pair, evaluated at the first pericenter passage. In all the previous sections, we have always referred our analysis to the pair of interacting galaxies: for example, the SFR is the total SFR, to which both galaxies of the pair contribute; the tidal parameter $T_{\mathrm{p}}$ has been evaluated following Eq. (10), thus summing the tidal effects suffered by the two galaxies. In this section, we will "decompose" our analysis, evaluating some physical quantities directly on each galaxy of the pair. This is in order to get a more detailed picture of the role played by each component in the evolution of the interacting system.

Being interested in explaining the relation found for mergers, we restrict our analysis here to the sample of merger galaxies, i.e. to all the galaxies involved in interactions that lead to mergers in over three Gyr of evolution.

First of all, for each galaxy in this sample, we evaluate the radius $r_{75}$ containing $75 \%$ of the total mass (gas+stars+dark matter) of the system, just after the pericenter passage with the companion, in order to quantify the expansion experienced by the galaxy, as an effect of the encounter. In Fig. $27, r_{75}$ is plotted against the tidal parameter $T_{\mathrm{p} \text {,gal }}$, which now is evaluated on the single galaxy and not on the pair. For simplicity, we have grouped galaxies into two subsamples: early-type systems, 
Mergers

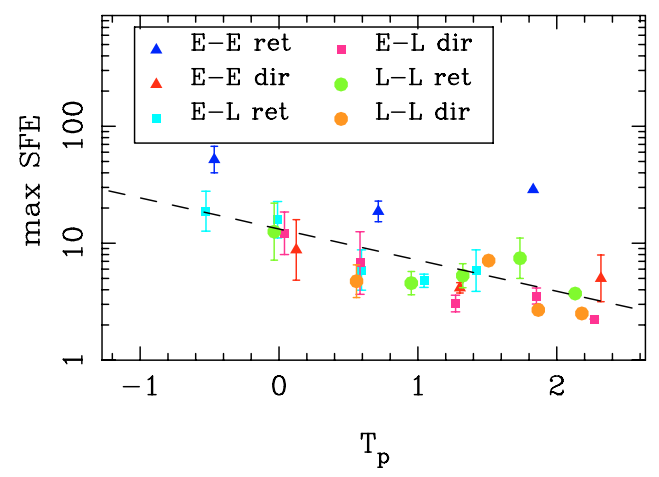

Flybys

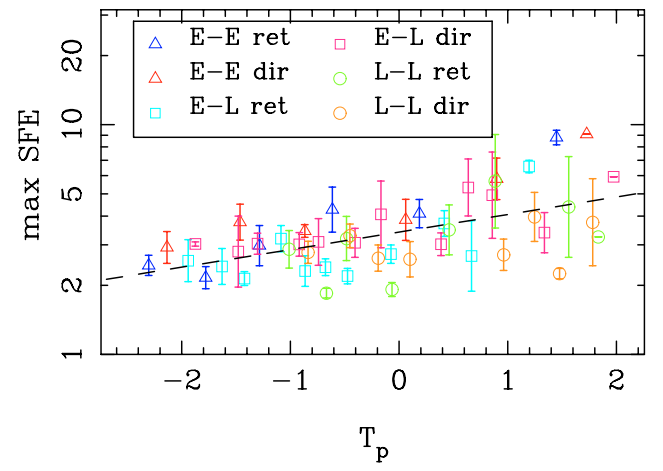

Fig. 25. Maximum star formation efficiency, relative to the isolated case, as a function of the tidal parameter $T_{\mathrm{p}}$ (see text), for flybys (lower panel) and mergers (upper panel). Different symbols correspond to different morphologies of interacting galaxies, as explained in the legends. The dashed lines represent the best linear least-square fits. Error bars represent the standard error of the mean.

including gE0 and gSa, and late-type ones, including gSb and gSd galaxies.

Not surprisingly, the figure shows a strong correlation between these two quantities, indicating that the stronger the interaction at the pericenter passage, the greater the subsequent expansion of the outer parts of the system. The radius $r_{75}$ is computed including all the galaxy components, but it is likely that a similar relation holds for the gaseous component only, in the sense that the gas mass ejected into tidal tails grows as tidal effects on the galaxy become stronger. This is shown in Fig. 28, where $M_{\text {gas,out }}$, the gas mass outside $20 \mathrm{kpc}$ from the galaxy center, is plotted against $T_{\mathrm{p}, \mathrm{gal}}$. $M_{\mathrm{gas}, \text { out }}$ has been evaluated just after the pericenter passage and it has been normalized to the gas mass present, at the same time, in the disk of the corresponding galaxy evolving alone.

To summarize, after the pericenter passage, the amount of gas in the galactic disk is depleted by two complementary effects:

1. an increase in the star formation rate in the disk, which accelerates the gas consumption;

2. the ejection of gas material from the disk into tidal tails.

The role these two phenomena play in depleting the gas mass in the disk is shown in Figs. 29 and 30.

The first figure shows $M_{\mathrm{gas}, \text { in }}$, the gas mass inside $20 \mathrm{kpc}$ from the galaxy center, versus $T_{\mathrm{p}, \mathrm{gal}}$. As in Fig. $28, M_{\text {gas, in }}$ has been evaluated just after the pericenter passage and it has been
Mergers

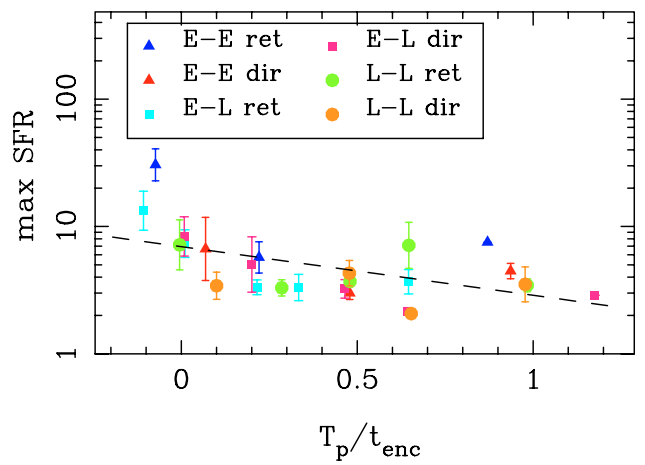

Flybys

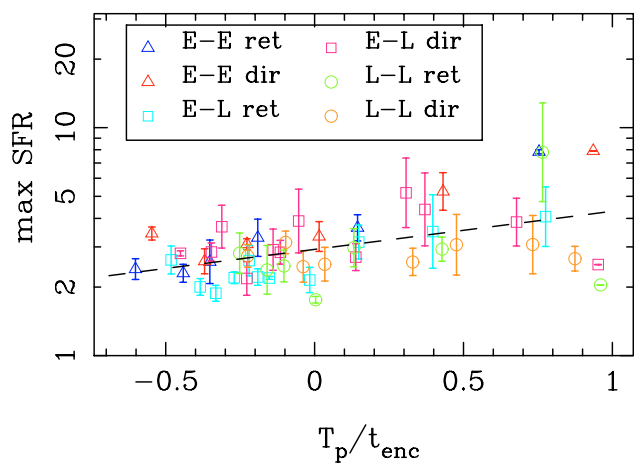

Fig. 26. Maximum star formation rate, relative to the isolated case, as a function of $T_{\mathrm{p}} / t_{\mathrm{enc}}$ (see text), for flybys (lower panel) and mergers (upper panel). Different symbols correspond to different morphologies of interacting galaxies, as explained in the legends. The dashed lines represent the best linear least-square fits. Error bars represent the standard error of the mean.

normalized to the gas mass present, at the same time, in the disk of the corresponding galaxy evolving alone.

The second figure shows the dependency on $T_{\mathrm{p}, \mathrm{gal}}$ of $M_{\mathrm{gas} \rightarrow \star}$, the integrated gas mass trasformed into star from the time $t=0$ to the time $t=t\left(R_{\mathrm{p}}\right)^{8}$. This quantity also has been normalized to the gas mass present, at the same time, in the disk of the corresponding galaxy evolving alone.

Comparing these two figures with Fig. 28, it is evident that usually the disk gas mass is decreased mainly because of the ejection of a copious gas fraction far out from the disk, while the encounter-enhanced star formation contributes only modestly to clear it out. Obviously, part of the gas mass lost in the tails, or acquired by the companion galaxy, can be reaccreted in the last stages of the merging event, thus contributing to the fuel available in the burst phase. But this is not the case for all the gas mass lost from the disk. In this sense, Fig. 28 helps to explain the trend found in Fig. 24 (top panel).

\subsection{Toward a formulation for the SFE}

For practical use, it is convenient to have a fitting formula for the estimation of the SFE, even if limited to planar encounters. The aim of this section is to propose a possible formulation, for mergers and flybys, as a function of: 1) the type of the encounter (E-E, E-L, L-L); 2) the orbital spin, if parallel or

\footnotetext{
${ }^{8}$ It is the time corresponding to the pericenter passage.
} 


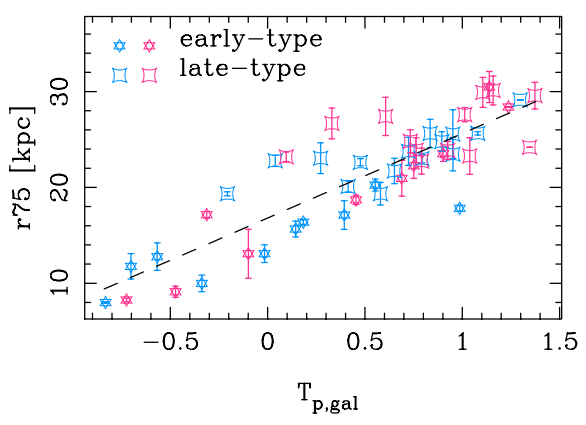

Fig. 27. Radius containing the $75 \%$ of the total (baryons+dark matter) mass of the galaxy versus the galaxy tidal parameter $T_{\mathrm{p}, \mathrm{gal}}$. Both quantities have been evaluated just after the first pericenter passage. Different symbols correspond to early and late-type galaxies, as explained in the figure. Red color refers to direct encounters, blue to retrograde ones. The analysis has been restricted to galaxies in the merger sample (see text). The dashed lines represent the best linear least-square fits. Error bars represent the standard error of the mean.

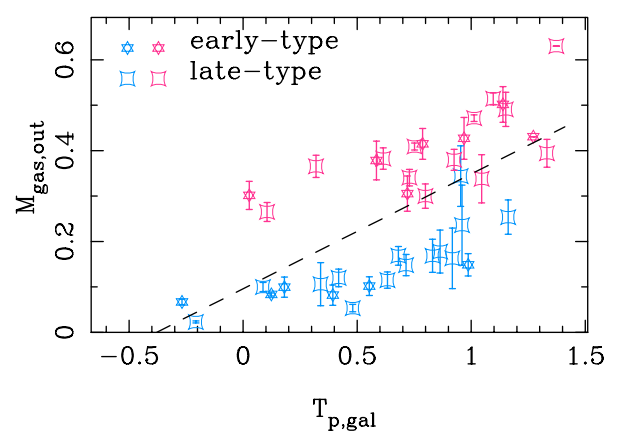

Fig. 28. Gas mass outside $20 \mathrm{kpc}$ from the galaxy center, versus the galaxy tidal parameter $T_{\mathrm{p}, \mathrm{gal}}$. $M_{\mathrm{gas}, \text { out }}$ has been evaluated just after the pericenter passage and it has been normalized to the gas mass present, at the same time, in the disk of the corresponding galaxy evolving alone. Different symbols in the figure correspond to early and late-type galaxies, as explained. Red color refers to direct encounters, blue to retrograde ones. The analysis has been restricted to galaxies in the merger sample (see text). The dashed lines represent the best linear least-square fits. Error bars represent the standard error of the mean.

antiparallel to the galaxies angular momentum (i.e. direct or retrograde orbit); 3 ) the pericenter distance $R_{2 \mathrm{~b}}$ of the Keplerian orbit of two points with masses equal to the masses of the interacting systems.

In other terms, we are looking for a function $f$ (morph, spin, $R_{2 \mathrm{~b}}$ ) so that

$\log _{10} \mathrm{SFE}_{\max }=f\left(\right.$ morph, spin, $\left.R_{2 \mathrm{~b}}\right)$.

As in the previous sections, for mergers and flybys, the maximum SFE is relative to that of the corresponding galaxies evolved in isolation.

In Sect. 4.7.4 we have shown the strong correlation existing between the maximum SFE and the tidal parameter $T_{\mathrm{p}}$ at the pericenter, giving a global fitting formula, which takes into account all the various morphological galaxy types. In general, this strong correlation between $T_{\mathrm{p}}$ and SFE still holds when grouping galaxies in different classes, depending on their morphological type and the orbital spin, direct or retrograde. This suggests using a right-hand side in Eq. (11) containing a parameter that could approximate $T_{\mathrm{p}}$. Note indeed that, for practical purposes, we are looking for a formulation for SFE that could be expressed only in terms of the initial parameters of the simulated encounter

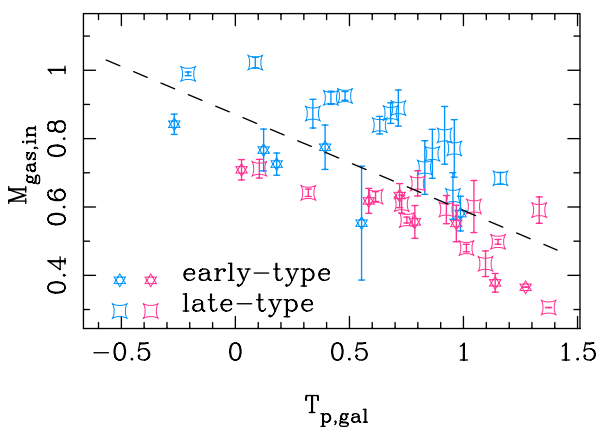

Fig. 29. Gas mass inside $20 \mathrm{kpc}$ from the galaxy center, versus the galaxy tidal parameter $T_{\mathrm{p}, \mathrm{gal}} . M_{\mathrm{gas}, \text { in }}$ has been evaluated just after the pericenter passage and it has been normalized to the gas mass present, at the same time, in the disk of the corresponding galaxy evolving alone. Different symbols in the figure correspond to early and late-type galaxies, as explained. Red color refers to direct encounters, blue to retrograde ones. The dashed lines represent the best linear least-square fits. Error bars represent the standard error of the mean.

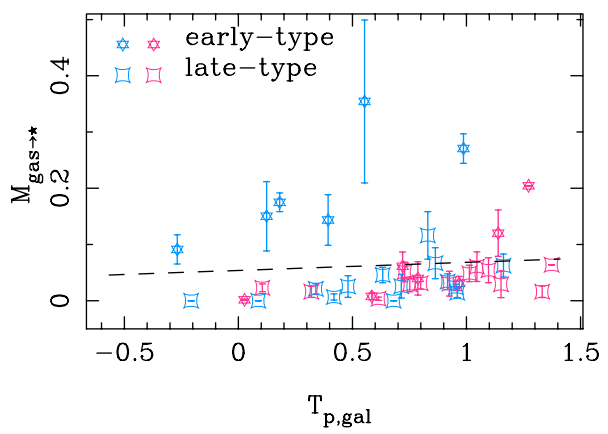

Fig. 30. Plot of $M_{\mathrm{gas} \rightarrow \star}$ versus the galaxy tidal parameter $T_{\mathrm{p}, \mathrm{gal}} . M_{\mathrm{gas} \rightarrow \star}$ represents the integrated gas mass transformed into star from the time $t=0$ to the time $t=t\left(R_{\mathrm{p}}\right)$. As in Figs. 28 and 29, this mass has been normalized to the gas mass present, at the same time, in the disk of the corresponding galaxy evolving alone. Different symbols in the figure correspond to early and late-type galaxies, as explained. Red color refers to direct encounters, blue to retrograde ones. Error bars represent the standard error of the mean. The analysis has been restricted to galaxies in the merger sample (see text).

and that does not depend on quantities that require an ad hoc simulation to be evaluated.

We remind the reader that the tidal parameter $T_{\mathrm{p}}$ at pericenter has been defined in Eq. (9) as the sum of the tidal parameters $T_{\mathrm{p}, 1}$ and $T_{\mathrm{p}, 2}$ relative to the two galaxies involved in the interaction, i.e.

$$
\begin{aligned}
T_{\mathrm{p}} & =\log _{10}\left[\frac{M_{2}}{M_{1}}\left(\frac{D_{1}}{R_{\mathrm{p}}}\right)^{3}\right]+\log _{10}\left[\frac{M_{1}}{M_{2}}\left(\frac{D_{2}}{R_{\mathrm{p}}}\right)^{3}\right] \\
& =\log _{10}\left[\left(\frac{D_{1} D_{2}}{R_{\mathrm{p}}{ }^{2}}\right)^{3}\right],
\end{aligned}
$$

$R_{\mathrm{p}}$ being the pericenter distance and $D_{1}$ and $D_{2}$ the galaxies scalelength. $R_{\mathrm{p}}$ was evaluated as the minimum distance between the two galaxy density centers and both $D_{1}$ and $D_{2}$ were approximated with the radius containing $75 \%$ of the total galaxy mass and were evaluated at the pericenter passage.

On the contrary, the suggested approximation for $T_{\mathrm{p}}$ will make use of all quantities yet available ab initio, i.e.

$T_{\mathrm{p}} \approx x=\log _{10}\left[\left(\frac{d_{1} d_{2}}{R_{2 \mathrm{~b}}{ }^{2}}\right)^{3}\right]$, 
Table 4. Values for the $A$ and $B$ constants in Eq. (15), for mergers and flybys.

\begin{tabular}{ccccc}
\hline \hline & \multicolumn{2}{c}{ Mergers } & \multicolumn{2}{c}{ Flybys } \\
\hline & $A$ & $B$ & $A$ & $B$ \\
\hline E-E ret & -0.098 & 1.557 & 0.135 & 0.653 \\
E-E dir & -0.126 & 0.826 & 0.115 & 0.724 \\
E-L ret & -0.202 & 1.089 & 0.078 & 0.482 \\
E-L dir & -0.098 & 0.779 & 0.024 & 0.543 \\
L-L ret & -0.121 & 0.954 & 0.027 & 0.461 \\
L-L dir & -0.026 & 0.621 & 0.004 & 0.467 \\
\hline
\end{tabular}

$R_{2 \mathrm{~b}}$ being the pericenter distance of the unperturbed Keplerian orbit of two bodies, having masses equal to the two galaxy masses, and $d_{1}$ and $d_{2}$ being the radii containing $75 \%$ of the total mass of the unperturbed galaxies.

The suggested formulation for the SFE is the following 9 :

$\log _{10} \mathrm{SFE}_{\max }=A \log _{10}\left[\left(\frac{d_{1} d_{2}}{R_{2 \mathrm{~b}}{ }^{2}}\right)^{3}\right]+B$,

being $A=A$ (morph, spin) and $B=B$ (morph, spin) constants depending on the morphology of the two galaxies involved in the interaction and on the orbital spin. Note that also the $x$ term depends on galaxy morphologies, via $d_{1}$ and $d_{2}$.

In Table $4, A$ and $B$ are given, both for mergers and flybys, and for different classes (E-E, E-L, L-L, in retrograde or direct orbits). Their values have been obtained performing a linear least-square fit between the $x$ values and the corresponding values of $\log _{10} \mathrm{SFE}_{\max }$ resulting from simulations.

A comparison between the curves

$y=A \log _{10}\left[\left(\frac{d_{1} d_{2}}{R_{2 \mathrm{~b}}{ }^{2}}\right)^{3}\right]+B$

and the "experimental" data for the SFE, for mergers and flybys, is shown in Figs. 31 and 32, respectively. The proposed formulation for the SFE reproduces the data quite well, with the modulus of the relative errors quite uniformly distributed with a mean value that is always below $30 \%$, with the only exception of early-late type direct mergers and late-late type retrograde flybys, where it is $\approx 50 \%$.

\subsection{Evolution in the $\left(\Sigma_{\text {gas }}, \Sigma_{S F R}\right)$ plane}

In the previous sections, we have seen that the star formation rate in the merging phase anticorrelates with the intensity of the tidal interaction at first pericenter passage and we explained this in terms of gas removal from the galactic disk of the two galaxies, just after the encounter. In this picture, the next step is to verify if the SFR during interactions and in the coalescence phase correlates with the quantity of gas material available in the galactic disk.

Several observations of normal galaxies suggest that the SFR on global scales is well represented by a Schmidt law of the form $\Sigma_{\mathrm{SFR}}=A \Sigma_{\text {gas }}{ }^{N}, \Sigma_{\text {gas }}$ being the gas density weighted inside the galactic optical radius and $\Sigma_{\mathrm{SFR}}$ the disk-averaged SFR surface density. The best fitting slope $N$, obtained with a conventional least squares fit, is about 1.4 (see Kennicutt 1998a). Despite the

9 The sample being composed only by giant galaxies, in the following formula no dependency on the galaxy masses is proposed. We refer the reader to following papers for a more general formulation, that could take into account also the mass ratio of the interacting galaxies.

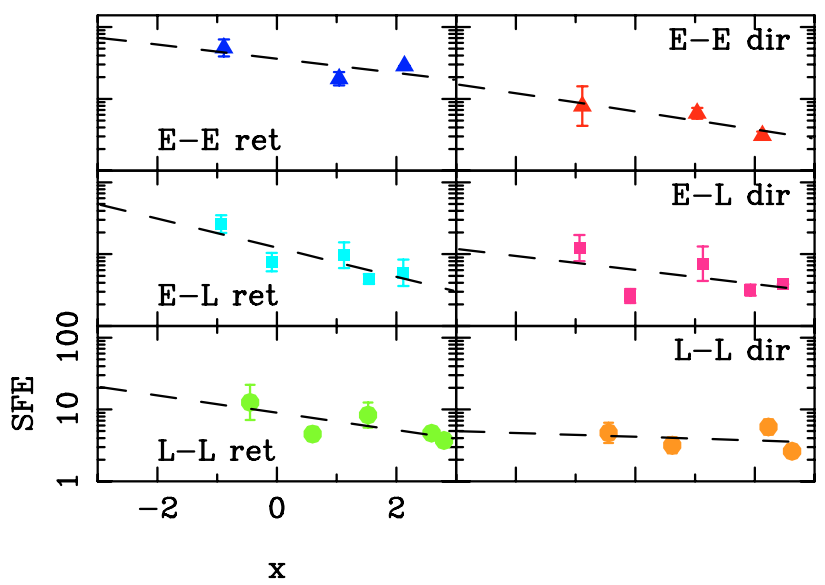

Fig. 31. SFE versus the parameter $x$ defined in Eq. (14) for mergers. Different panels refer to different classes for the encounters, depending on the morphology of the interacting galaxies and on the orbital spin. Error bars represent the standard error of the mean. The dashed line in each panel represents the proposed formulation for the SFE (see Eq. (15)).

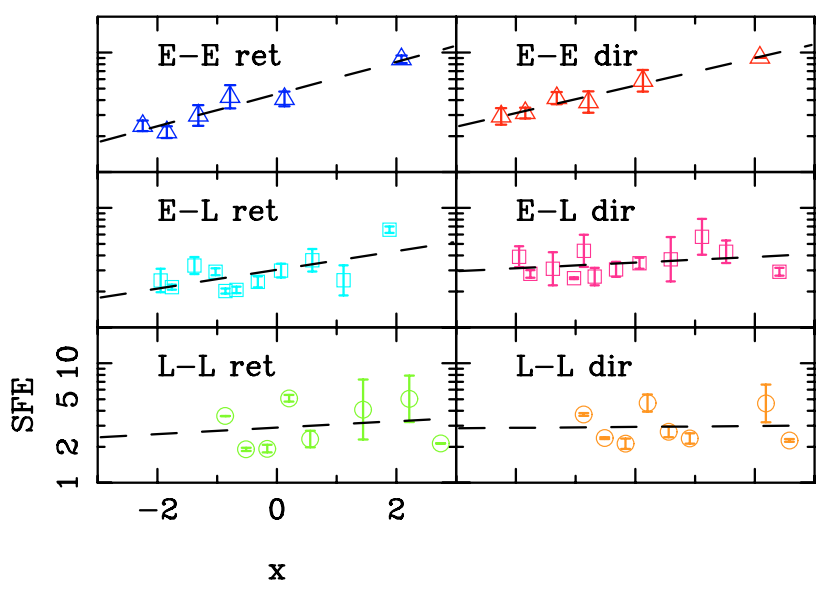

Fig. 32. SFE versus the parameter $x$ defined in Eq. (14) for flybys. Different panels refer to different classes for the encounters, depending on the morphology of the interacting galaxies and on the orbital spin. Error bars represent the standard error of the mean. The dashed line in each panel represents the proposed formulation for the SFE, described (see Eq. (15)).

large variation for a given value of $\Sigma_{\text {gas }}$, these data show that a correlation between SFR and gas density exists, which is also largely independent of galaxy type. A similar correlation, with a comparable $N$ slope, exists for starburst galaxies, if averaging the SFR density and the gas density on the inner (1-2 kpc) galactic region, both the $\Sigma_{\text {gas }}$ and the $\Sigma_{\text {SFR }}$ values being in this case 1-4 orders of magnitude higher than those obtained for normal galaxies, thus suggesting that a large amount of gas is present in the central region to sustain the burst activity.

Other authors have found some variations in the value of the $N$ slope: Wong \& Blitz (2002) investigating a sample of seven molecule rich spiral galaxies found $N \sim 1.1-1.7$, depending on the correction for extinction in $\mathrm{H} \alpha$ emission, in deriving the SFR; Boissier et al. (2003) found $N \sim 2.0$ for a sample of sixteen spiral galaxies; finally Gao \& Solomon (2004) suggested a star formation law with a power-law index of 1.0 in terms of dense molecular gas content, when studying a sample 


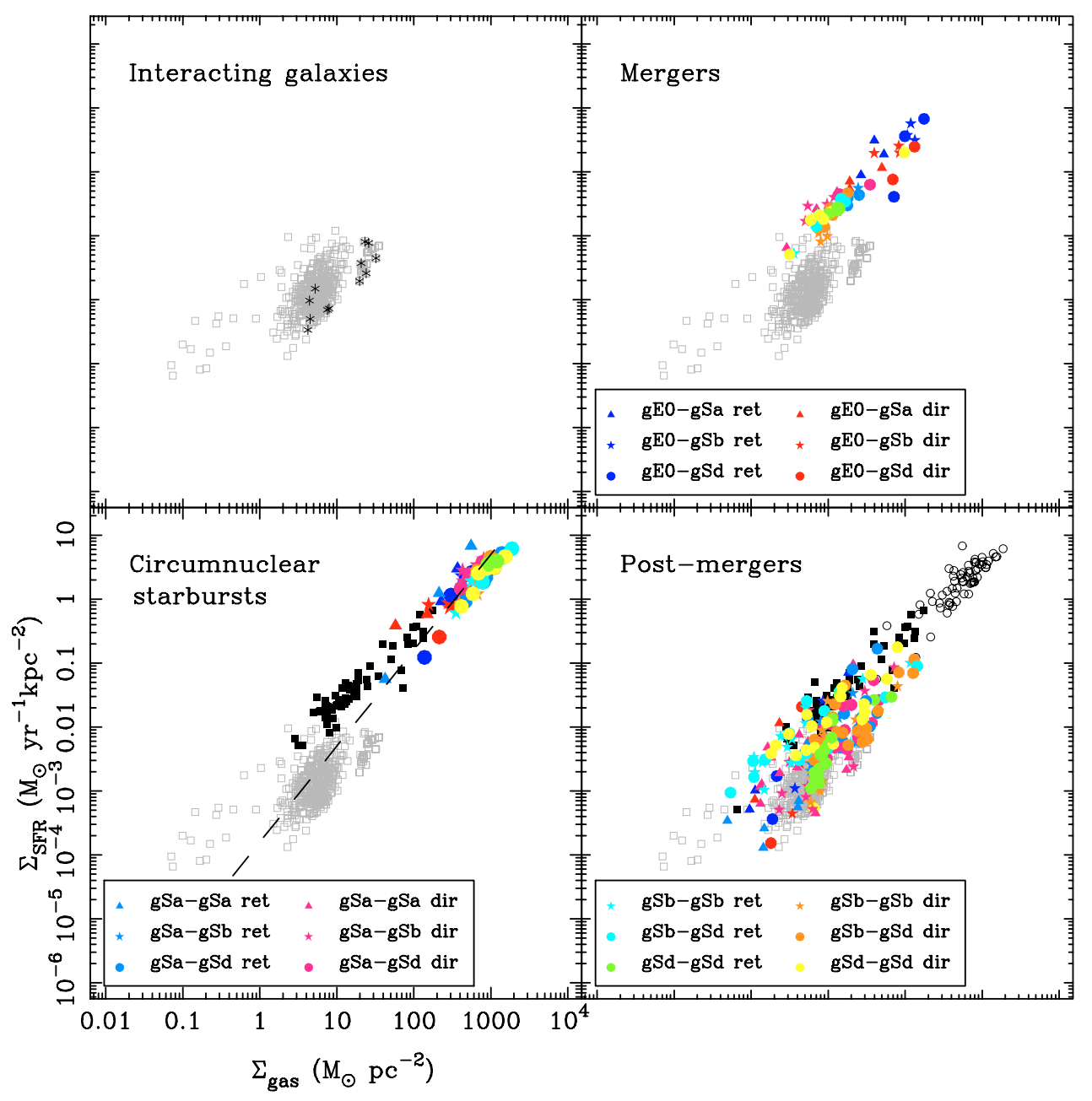

Fig. 33. The global Schmidt law in galaxies. Upper left panel: $\left(\Sigma_{\text {gas }}, \Sigma_{\mathrm{SFR}}\right)$ relation for interacting galaxies (grey empty squares). For comparison, also isolated galaxies are shown (stars). Upper right panel: $\left(\Sigma_{\text {gas }}, \Sigma_{\mathrm{SFR}}\right)$ relation for merging galaxies. Different symbols in this and in the next two panels are explained in the legends. For comparison, the interacting galaxy sample is shown with grey empty squares. Lower left panel: circumuclear starburst. For comparison, interacting galaxies (grey empty squares) and mergers (black squares) are also shown. The dashed line represent a "global" Schmidt star formation law of the form $\Sigma_{\mathrm{SFR}} \propto \Sigma_{\mathrm{gas}}{ }^{1.4}$ (Kennicutt 1998a), and it is shown for a comparison with the data. Lower righ panel: $\left(\Sigma_{\text {gas }}, \Sigma_{\mathrm{SFR}}\right)$ relation for post-merger galaxies. For comparison, interacting galaxies (grey empty squares), mergers (black squares) and circumnuclear starburst (black empty circles) are also shown.

of 65 normal spirals, luminous infrared and ultraluminous infrared galaxies.

In this framework, it is interesting to see whether our sample obeys a similar global relation, provided that on local scales our star formation recipe is a Schmidt type. In particular, we are interested in finding out if interacting and starburst galaxies follow a "global" Schmidt law of the type $\Sigma_{\mathrm{SFR}}=A \Sigma_{\text {gas }}{ }^{N}$, as found observationally for normal spiral galaxies (Kennicutt 1998a) and if, more generally, it is possible to trace a global evolution on the $\Sigma_{\text {gas }}-\Sigma_{\text {SFR }}$ plane, for pre-interaction, interacting, starburst galaxies and mergers.

To compute $\Sigma_{\text {gas }}$ and $\Sigma_{\text {SFR }}$, we evaluated the SFR and the gas amount inside a radius containing $85 \%$ of the total visible (gas+stars) galaxy mass ${ }^{10}$ and then we calculated the diskdensity relative quantities. For interacting galaxies, we evaluated the two quantities for each galaxy of the pair, while for starburst,

${ }^{10}$ Note that for interacting and starburst galaxies, due to the fact that part of the mass populates tidal tails, thus spreading for several hundreds $\mathrm{kpc}$ outside the galactic center, we restricted our analysis to $85 \%$ of the total visible (gas+stars) mass located inside $20 \mathrm{kpc}$ from the galaxy center. circumnuclear starbursts and post-merger $\Sigma_{\text {gas }}$ and the $\Sigma_{\mathrm{SFR}}$ are evaluated on the resulting merger.

The main findings of our analysis are summarized in Fig. 33, which shows different galaxy samples (interacting, starbursts, circumnuclear starbursts and mergers $)$ in the plane $\left(\Sigma_{\mathrm{gas}}, \Sigma_{\mathrm{SFR}}\right)$.

In this plane the interacting galaxies (upper left panel) lie in a region which extends over two orders of magnitude both in $\Sigma_{\text {gas }}$, from 0.1 to $30 M_{\odot} \mathrm{pc}^{-2}$, and in $\Sigma_{\mathrm{SFR}}$ $\left(10^{-4}-0.02 M_{\odot} \mathrm{yr}^{-1} \mathrm{kpc}^{-2}\right)$. No obviously different behavior is found for different morphological types, i.e. for early- and latetype systems. This sample includes galaxies at very different stages of interactions: there are systems that are well before the first close passage, hundreds of kpc distant from the companion (in this sense they can be considered as isolated) and systems close to the merging phase. We eliminated from this sample (as in the isolated sample shown in the same panel for comparison) the transient initial burst phase, corresponding to the emergence of density waves in the galactic disks (see Figs. 6 and 7). Note that the best linear fit reported in Fig. 33 for interacting galaxies would be steeper if the low $\Sigma_{\text {gas }}-\Sigma_{\mathrm{SFR}}$ values in this sample were not considered. 
The starburst phase is represented in the upper right panel. The high correlation found for this sample (0.9) is remarkable. We should point out that, in this phase, galaxies occupy an extended region in $\Sigma_{\text {gas }}$, and reach higher levels of SFR density, with respect to interacting pairs. Note also that mergers resulting from encounters between an elliptical and a spiral have, in general, higher gas surface densities and a higher star formation rate density with respect to spiral-spiral mergers. The situation changes if one restricts the analysis of starburst galaxies to circumnuclear regions, in the inner kpc region of the galaxy. In this case (lower left panel) the nature of the starburst event clearly emerges: the gas circumnuclear density is, on average, at least 100 times higher than that of the overall disk, thus giving rise to a strongly enhanced SFR, whose circumnuclear density is far greater than those of interacting and starburst galaxies as a whole. In this case, late-type spiral encounters show higher SFR densities, when compared to mergers involving an earlytype system.

Once the starburst phase is over, the mergers evolve toward the lower left part of the $\left(\Sigma_{\text {gas }}, \Sigma_{\text {SFR }}\right)$ plane (as shown in the lower right panel in Fig. 33), having at this point used up most of their gas reservoirs.

This overall trend in the location of normal and starburst galaxies in the $\left(\Sigma_{\text {gas }}, \Sigma_{\mathrm{SFR}}\right)$ plane is also confirmed observationally (see Kennicutt 1998a, Fig. 9 left). Note that in this case highest values (for normal and merging galaxies) are reached both in the $x$ and $y$ axis, but that this is merely due to the fact that our simulations start with initial conditions typical of present day galaxies and then evolve over three Gyr in time, so that the amount of gas in the simulated systems is only initially comparable to that of galaxies in the local Universe. In particular, the position of isolated and interacting galaxies in Fig. 33 top left is a reflection of gas amount.

The present study on the evolution of isolated, interacting and merging galaxies in the $\left(\Sigma_{\text {gas }}, \Sigma_{\mathrm{SFR}}\right)$ plane extends and enriches earlier numerical research concerning star formation in isolated galaxies and in major interactions. Springel (2000), for example, studied the composite Kennicutt law for a small set of simulated isolated and merging galaxies, showing that his sample corresponds remarkably closely to the global Schmidt law over a large dynamic range. But the accordance of the data with the global Schmidt law is a natural consequence of the chosen star formation and feedback recipes he adopted, being the free parameters chosen in order to satisfy Kennicutt's findings.

In an extensive study conducted to analyze how star formation depends on the adopted parametrization for feedback, Cox et al. (2006) showed that isolated galaxies satisfy the empirical Kennicutt law, when including a gas density threshold for star formation. Indeed, when averaging star formation rate and gas surface density within azimuthal annulli, and adopting feedback parameters that were not too high, they found a good accordance with the results in Fig. 3 in Kennicutt (1998b). Then, studying the Kennicutt law for the merger sample, using an azimuthal aperture of radius $2 \mathrm{kpc}$, they confirmed that the interacting sample also closely tracks the empirical star formation law.

But both these studies use a star formation and feedback modelization designed to reproduce the empirical Kennicutt law. In this sense, the present study investigates the $\Sigma_{\text {gas }}-\Sigma_{\text {SFR }}$ correlation, in a more general context. Indeed, starting from a star formation recipe based on the local gas volume density (Eq. (5)), it is not immediately clear that a global (on 10-20 kpc scales) relation, based on gas surface density, must hold. Evidently, we do not claim to reproduce the slope found for the empirical law, but, nevertheless, it is striking to see how a global (i.e. on kpc scales)
$\Sigma_{\text {gas }}-\Sigma_{\text {SFR }}$ relation is satisfied, for isolated, interacting, merging and post-merger galaxies.

\subsection{Gas inflow}

It is well established that the interaction with a companion usually destabilises the disk of a galaxy, which develops nonaxisymmetric structures (such as spiral arms and bars), responsible for exerting torques on the gas material (Noguchi 1988; Barnes \& Hernquist Barnes \& Hernquist 1996; Mihos \& Hernquist Mihos \& Hernquist 1996; Combes Combes 2001). In this way, the gas loses angular momentum and falls into the inner galactic regions, where a starburst takes place (see Smith et al. 2007, for an observational study of 35 interacting galaxy pairs, that show gas concentration in the inner regions, with a subsequent enhancement of the central star formation rate).

In the previous section, we have shown indeed that the star formation enhancement in the merging phase is a strongly inhomogeneous process, taking place mostly in the central kiloparsec. Here we want to describe in greater detail the gas dynamics during the different phases of the encounter. To do this, we studied the occurence and evolution of asymmetries in the disk stellar distribution and the response of the gas component to their emergence. With this in mind we first Fourier-analyzed the disk surface density distribution, focusing in particular on the amplitudes of the Fourier components $m=1$ and $m=2$. Then we studied the evolution of the gas lagrangian radii containing $10 \%$, $25 \%, 50 \%$ and $75 \%$ of the total gas mass, in order to have a picture of gas inflows into the central regions. Obviously, it is not possible to describe here the results of all the different encounters simulated, so we will describe in detail only two of the cases analyzed.

To Fourier-analyze the disk surface density distribution, we adopted the following procedure:

- firstly, the angular momentum of the disk galaxy was evaluated and the galactic plane was rotated as a function of this, in order to have the galactic spin parallel to the $z$-axis of the reference frame;

- then the $x-y$ plane was divided into an annular grid (exponentially spaced in radius and linearly in azimuth) between the center $C$ of the disk galaxy and $10 \mathrm{kpc}$.

- the surface mass density distribution on the $x-y$ plane was evaluated, taking into account only the old galactic stellar component that lies between \pm 500 pc from the galactic disk.

- once the density $\Sigma(r, \theta)$, being $r$ the distance from the galaxy center and $\theta$ the azimuth, had been computed, for each annular ring, we fitted $\Sigma(r, \theta)$ with the following function

$$
\begin{aligned}
\frac{f(\theta)}{A_{0}}= & 1+\left[A_{1} \sin \left(\theta+\phi_{1}\right)+A_{2} \sin \left(2 \theta+\phi_{2}\right)\right. \\
& \left.+\ldots .+A_{8} \sin \left(8 \theta+\phi_{8}\right)\right]
\end{aligned}
$$

obtaining a value of $A_{0}, A_{1}, \ldots, A_{8}$ and $\phi_{1}, \ldots, \phi_{8}$ for each annular ring.

- finally, for each configuration, we evaluated the averaged value $\left\langle A_{1}\right\rangle$ and $\left\langle A_{2}\right\rangle$ of $A_{1}$ and $A_{2}$ respectively, between 1 and $10 \mathrm{kpc}$ and we adopted these values to quantify the "strength" of the $m=1$ and $m=2$ asymmetries.

The results of this analysis are shown in Fig. 34, which refers to two encounters (a direct and a retrograde one) between an elliptical and a gSb galaxy. In the first column, the relative distance between the two interacting system is shown, as a function of time, while the second and third column show the evolution of 

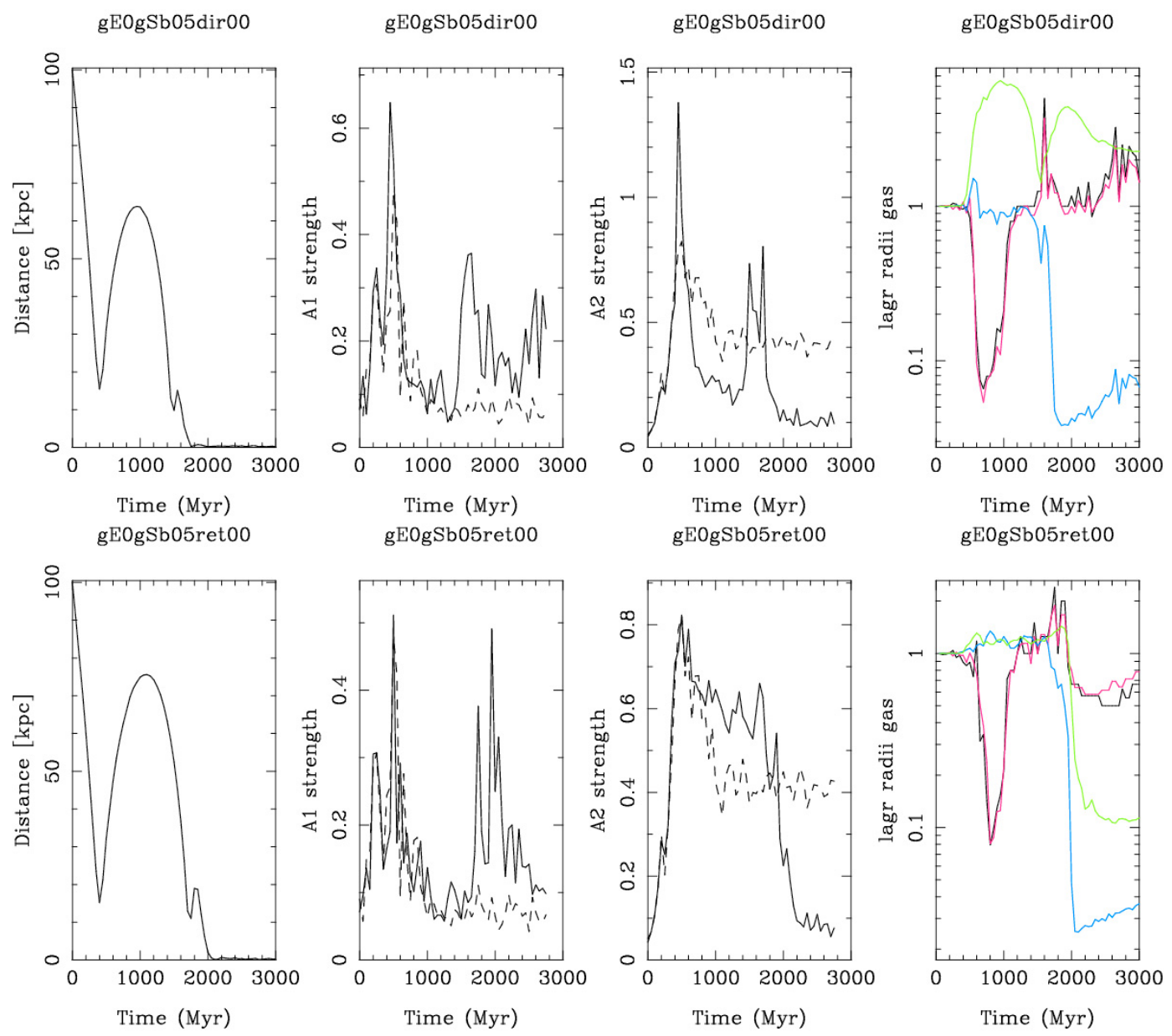

Fig. 34. Gas dynamics during the interaction and subsequent merger between an elliptical and a spiral Sbc galaxy. The first row refers to the two galaxies in direct orbit, the second row to galaxies in retrograde interaction. First column: relative distance between the two galaxy centers versus time. Second column: evolution of the $A_{1}$ strength, versus time (solid line). For comparison, the evolution of the $A_{1}$ asymmetry of the same galaxy evolving alone is shown (dotted line). Third column: evolution of the $A_{2}$ strength, versus time (solid line). For comparison, the evolution of the $A_{2}$ asymmetry of the same galaxy evolving alone is shown (dotted line). Fourth column: evolution with time of gas lagrangian radii containing $10 \%$ (black), $25 \%$ (red), $50 \%$ (blue) and 75\% (green) of the gas mass are shown, in units of the corresponding gas radii of the isolated gSb galaxy.

$m=1$ and $m=2$ asymmetries, respectively. It is evident from this plot that:

- tidal encounters usually amplify both $m=1$ and $m=$ 2 asymmetries;

- at the first pericenter passage or during flybys, galaxies in direct orbits usually develop $A_{2}$ asymmetries more pronounced than those arising in retrograde encounters;

- for mergers, a second amplification in the $A_{1}$ and $A_{2}$ asymmetries, relative to the isolated case, occurs when the two galaxies are in the final stage of coalescence;

- in the phase between first pericenter passage and merger, the $A_{1}$ and $A_{2}$ values can be either higher or lower than those of the same galaxies evolving in isolation.

The emergence of strong asymmetries in the disk obviously influences the gas dynamics, as shown in the last column of Fig. 34, where the lagrangian radii for the gas component are shown ${ }^{11}$. Indeed, after the first pericenter passage, as the disk galaxy develops transient $m=1$ and $m=2$ asymmetries, an initial gas inflow, involving about $25 \%$ of the gas mass takes place, accompanied by an increase in the star formation efficiency. This inflow lasts for about $200 \mathrm{Myr}$ and is followed by a re-expansion

${ }_{11}$ In fact here we refer to the gas+new stars component. of the inner gas lagrangian radii, probably due to an enhancement in the gas kinetic energy from SNe explosions. But the most dramatic and very rapid gas compression starts only when the two interacting galaxies are in the final phase of the merger: in this case the rapidly changing galactic potential causes the inflow of a substantial fraction of the gas mass (between 50\% and $80 \%$ of the gas mass involved) into the inner kiloparsec region, where a strong starburst takes place.

\section{Discussion: are galaxy interactions always starburst triggers?}

In the previous section, we have looked at the evolution of the star formation rate during flybys and mergers in detail, showing the great variety of SFR evolutions occurring during galaxy interactions and presenting an in-depth study of the SFR dependency on several parameters. We have already pointed out (Sect. 4.5) that interactions are not always a sufficient condition to convert high gas mass quantities into new stars. Here we wish to go into the discussion on this point in greater depth, because of its potential impact on observational studies as well. Evidently, we are bearing in mind that the statistical analysis presented in this paper has its limitations, which we will try to remove in later research. For example, even though we were exploring a whole 
Hubble sequence (from early-type to late-type giant galaxies), all the simulations were carried out using a unique dark matter model. Having shown in Sect. 4.8 that tidal effects are crucial in determining the SFR in the merging phase, it could be interesting to check, in subsequent work, how the overall analysis depends on the dark matter model adopted (varying the density profiles, the limiting radii, the central concentrations, and so on).

The present work is also limited to coplanar encounters, with galaxies having null relative disk inclinations, and spins parallel to the orbital angular momentum.

Notwithstanding this the large sample (216) of interacting galaxies presented here is yet sufficient to draw some preliminary conclusions concerning the interaction-starburst connection.

Figure 35 shows the histogram of the maximum SFR for mergers and flybys. As in the previous section, this value is relative to the SFR of the galaxy evolved in isolation. The plot clearly highlights that mergers are not always starburst triggers: indeed the number of mergers that produce star formation rates ten times higher than those of isolated galaxies are about $17 \%$ of the total merger sample, while half of the sample shows only a moderate enhancement in the SFR (less than four times the isolated case).

High SFR are not only less frequent, but are also characterized by the shortest duration times. This is shown in Fig. 36, where the histogram of the burst duration is plotted, for two different SFR enhancement levels. About 36\% of the whole interacting galaxies sample ${ }^{12}$ sustains SFRs two times higher than those of the isolated counterparts for a time greater than 500 Myr. But this duration significatively diminishes, for galaxies whose relative SFR is greater than five. In this case, the maximum starburst duration is less than $600 \mathrm{Myr}$ and only $13 \%$ of the whole galaxy sample sustains a SFR enchancement greater than five for more than $100 \mathrm{Myr}$ (small window in Fig. 36). Lastly, galaxies that show SFRs 20 times higher than those of the isolated counterparts are able to substain it for $150 \mathrm{Myr}$, in the most favourable cases.

Not only is the starburst frequency low, but also the integrated star formation rate, compared to the isolated case, seems to support the idea that interactions are not always sufficient to convert high gas mass quantities into new stars, as the histogram in Fig. 37 shows. Flybys and mergers can produce twice as many stars as isolated galaxies, but about $50 \%$ of all the mergers, and $76 \%$ of flybys, have an ISFR (see Sect. 4.3.1 for its definition) that is only 1.25 times greater than that of isolated galaxies.

\section{Conclusions}

We have investigated the enhancement of the star formation rate in galaxy interactions by numerical simulations, comparing the star formation properties of more than two hundred pairs of interacting and merging galaxies with those of isolated galaxies.

The present research partially confirms previous numerical investigations (Mihos \& Hernquist 1994a,b, 1996; Springel 2000; Cox et al. 2006), in the sense that major galaxy interactions and mergers can trigger strong nuclear starburst, but it shows clearly that this is not always the case, i.e. mergers are not always starburst triggers and galaxy interactions are not a sufficient condition for converting large quantities of gas mass into new stars. This is mainly because strong tidal interactions at the first pericenter passage can remove a large amount of gas from

\footnotetext{
12 i.e. including flybys and mergers together.
}

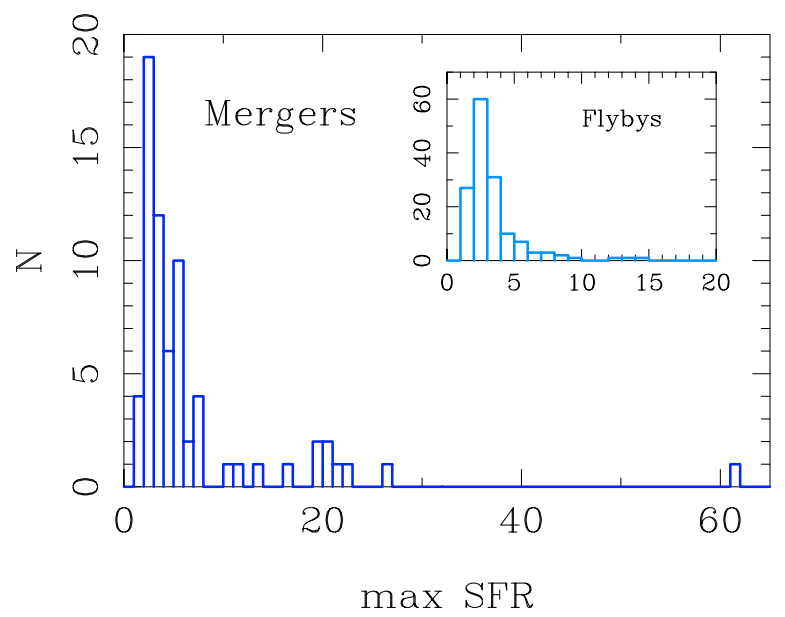

Fig. 35. Histograms of the maximum SFR (relative to the isolated case), for mergers. Flybys are shown for comparison in the small window inserted in the figure.

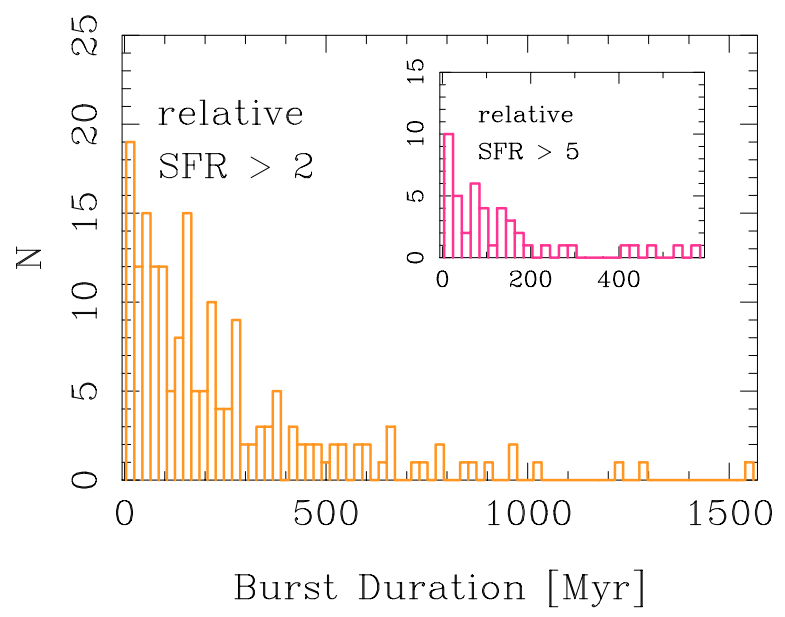

Fig. 36. Histograms of duration of enhanced SFR, for the whole sample of interacting galaxies (mergers and flybys). Two thresholds are shown: relative $S F R>2$ and relative $S F R>5$ (the latter is shown in the small window inserted in the figure).

the galaxy disks. This gas material, ejected into the tidal tails, is only partially re-acquired by the galaxies in the last phase of the merging event.

We have shown that the star formation rate in the merging phase:

1. does not depend mainly on the total amount of gas mass available just before the final coalescence phase, this total amount of gas content being the sum of that present in the tails and in the main body of the system.

In turn, it is strongly negatively correlated with:

2. the distance between the two galaxy centers at the first pericenter passage, in the sense that, on average, galaxies that pass too close to one another also produce the lowest bursts of star formation;

3. the amplitude of the tidal forces at pericenter, i.e. pairs that suffer less intense tidal actions at the first passage are able to preserve a great mass fraction in the disk, which constitutes the fuel for the nuclear starburst in the merging phase. Furthermore,

4. the enhancement of the star formation in the merging phase depends on the galaxy spin, in the sense that, on average, 


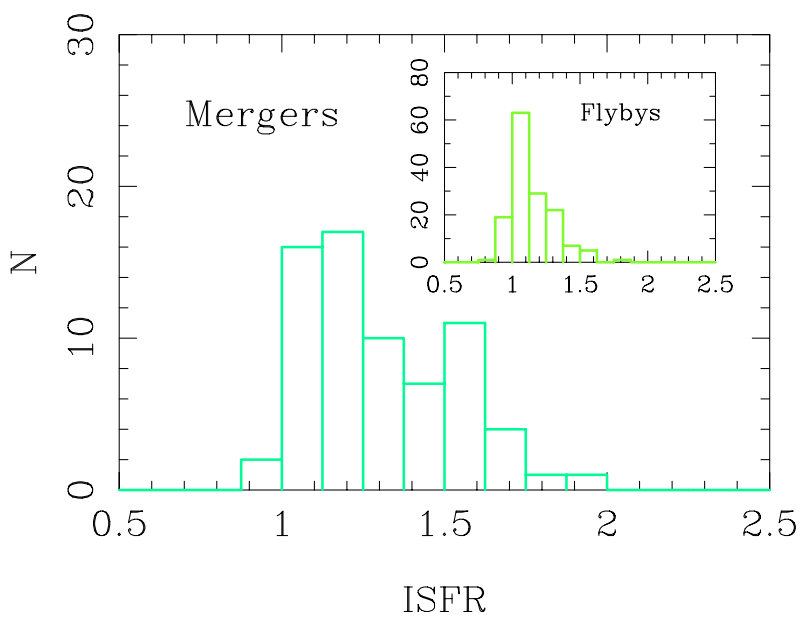

Fig. 37. Histogram of the relative ISFR, for mergers. Flybys are shown for comparison in the small window inserted in the figure.

galaxies in retrograde orbits are stronger starburst triggers than those involved in direct encounters.

We have also analyzed the evolution in the $\left(\Sigma_{\text {gas }}, \Sigma_{\mathrm{SFR}}\right)$ plane of interacting, mergers and post-merger galaxies, finding that, globally, the Kennicutt-Schmidt law applies statistically for all the different stages of interaction.

Finally, we have put forward a formulation for the SFE at the pericenter passage for flybys and in the coalescence phase for mergers. The general laws we derived depend only on the main parameters of the encounters, such as the orbital spin, the pericenter separation and the galaxy dimensions.

Obviously, it is still necessary to use a larger range of parameters, for example to get an insight into the dependency of the star formation efficiency on the orientation of the galaxy disks with respect to the orbital plane, or on the mass ratio of the galaxies in the pair. It would be interesting also to perform a subset of these simulations while varying the star formation rule, i.e. from a density-dependent, Schmidt-type one, to a formulation that could take into account energy dissipation in shocks, as proposed by Barnes (2004). Whatever the case may be, we believe that this research can contribute, on the one hand, to clarifying the physical mechanisms behind the large range of star formation enhancements found in observed interacting pairs, while furnishing, on the other hand, simple star formation formulations for theoretical modelling.

Acknowledgements. P. D. M. thanks C. Jog for useful discussions and suggestions, and Y. Revaz for providing the python parallelized pNbody package (see http://aramis.obspm.fr/ revaz/pNbody/index.html), used for making galaxy maps.

The authors are deeply grateful to F. Bournaud, for a careful reading of a first version of this paper, all his suggestions having undoubtfully improved the quality of the manuscript and the presentation of the results.

We wish to thank an anonymous referee for his/her comments, which helped in improving the contents of this paper.

This research used computational resources of the Informatic Division of the Paris Observatory, and those available within the framework of the Horizon Project (see http: //www . projet-horizon. fr/).

\section{References}

Barnes, J. 2004, MNRAS, 350, 798

Barnes, J., \& Hut, P. 1986, Nature, 324, 446

Barnes, J. E., \& Hernquist, L. 1996, ApJ, 471, 115

Bergvall, N., Laurikainen, E., \& Aalto, S. 2003, A\&A, 405, 31

Binney, J., \& Tremaine, S. 1987, Galactic Dynamics (Princeton: Princeton Univ. Press)

Boissier, S., Prantzos, N., Boselli, A., et al. 2003, MNRAS, 346, 1215

Bournaud, F., Combes, F., Jog, C. J., et al. 2005, A\&A, 438, 507

Casasola, V., Bettoni, D., \& Galletta, G. 2004, A\&A, 422, 941

Casertano, S., \& Hut, P. 1985, ApJ, 298, 80

Cimatti, A., Pozzetti, L., Mignoli, M., et al. 2002, A\&A, 391, L1

Cox, T. J., Primack, J., Jonsson, P., et al. 2004, ApJ, 607, L87

Cox, T. J., Jonsson, P., Primack, J., et al. 2006, MNRAS, 373, 1013

Combes, F. 2001, in Advanced Lectures on the Starburst-AGN, ed. I. Aretxaga, D. Kunth, \& R. Mújica (Singapore: World Scientific), 223 [arXiv: astro-ph/0010570]

Combes, F. 2004, in Penetrating Bars Through Masks of Cosmic Dust: The Hubble Tuning Fork Strikes a New Note, ed. D. L. Block et al. (Dordrecht: Kluwer), 57 [arXiv: astro-ph/0406306]

Combes, F., Prugniel, P., Rampazzo, R., et al. 1994, A\&A, 281, 725

Combes, F., García-Burillo, S., Braine, J., et al. 2006, A\&A, 460L, 49

Cullen, H., Alexander, P., Green, D. A., et al. 2006

[arXiv: astro-ph/0611617]

Devriendt, J., \& Guiderdoni, B. 2000, A\&A, 363, 851

Flores, H., Hammer, F., Elbaz, D., et al. 2004, A\&A, 415, 885

Gabbasov, R. F., Rodríguez-Meza, M. A., Klapp, J., et al. 2006, A\&A, 449, 1043

Gao, Y., \& Solomon, P. M. 2004, ApJ, 606, 271

Georgakakis, A., Forbes, D. A., \& Norris, R. P. 2000, MNRAS, 318, 124

Gingold, R. A., \& Monaghan, J. J. 1982, 1982JCoPh, 46, 429

Governato, F., Mayer, L., Wadsley, J., et al. 2004, ApJ, 607, 688

Harfst, S., Theis, C., \& Hensler, G. 2006, A\&A, 449, 509

Hernquist, L. 1993, ApJS, 86, 389

Hernquist, L., \& Katz, N. 1989, ApJS, 70, 419

Jungwiert, B., Combes, F., \& Palous, J. 2001, A\&A, 376, 85

Kapferer, W., Knapp, A., Schindler, S., et al. 2005, A\&A, 438, 87

Katz, N. 1992, ApJ, 391, 502

Kauffmann, G., \& Charlot, S. 1998, MNRAS, 297, L23

Kauffmann, G., \& Haehnelt, M. 2000, MNRAS, 311, 576

Kennicutt, R. C., Jr. 1983, ApJ, 272, 54

Kennicutt, R. C., Jr. 1998a, ARA\&A, 36, 189

Kennicutt, R. C., Jr. 1998b, ApJ, 498, 541

Kennicutt, R. C., Jr., Tamblyn, P., \& Congdon, C. E. 1994, ApJ, 435, 22

Kennicutt, R. C., Calzetti, D., Walter, F., et al. 2005, AAS, 207, 6314

Le Fèvre, O., Abraham, R., Lilly, S. J., et al. 2000, MNRAS, 311, 565

Lilly, S. J., Le Fevre, O., Hammer, F., et al. 1996, ApJ, 460, L1

Lucy, L. B. 1977, AJ, 82, 1013

Madau, P., Ferguson, H. C., Dickinson, M. E., et al. 1996, MNRAS, 283, 1388

Meza, A., Navarro, J. F., Steinmetz, M., et al. 2003, ApJ, 590, 619

Mihos, C., \& Hernquist, L. 1994a, ApJL, 431, 9

Mihos, C., \& Hernquist, L. 1994b, ApJ, 437, 611

Mihos, C., \& Hernquist, L. 1996, 464, 641

Naab, T., Jesseit, R., \& Burkert, A. 2006, MNRAS, 372, 839

Nagamine, K., Springel, V., Hernquist, L., et al. 2004, MNRAS, 350, 385

Noguchi, M. 1988, A\&A, 203, 259

Roberts, M. S., \& Haynes, M. P. 1994, ARA\&A, 32, 115

Smith, B. J., Struck, C., Hancock, M., et al. 2007, AJ, 133, 791

Solomon, P. M., \& Sage, L. J. 1988, ApJ, 334, 613

Somerville, R. S., Primack, J. R., \& Faber, S. M. 2001, MNRAS, 320, 504

Sommer-Larsen, J., Götz, M., \& Portinari, L. 2003, ApJ, 596, 47

Springel, V. 2000, MNRAS, 312, 859

Springel, V., \& Hernquist, L. 2003, MNRAS, 339, 312

Steidel, C. C., Adelberger, K. L., Giavalisco, M., et al. 1999, ApJ, 519, 1

Steinmetz, M., \& Müller, E. 1994, A\&A, 281, L97

Tissera, P. B. 2000, ApJ, 534, 636

Tissera, P. B., Dominguez-Tenreiro, R., Scannapieco, C., et al. 2002, MNRAS, 333, 327

Wang, Z., Fazio, G. G., Ashby, M. L. N., et al. 2004, ApJS, 154, 193

Wong, T., \& Blitz, L. 2002, ApJ, 569, 15 